\title{
Bayesian Models of Interval Timing and Distortions in Temporal Memory as a Function of Parkinson's Disease and Dopamine-Related Error Processing
}

\author{
Bon-Mi Gu*, Anita J.Jurkowski**, Jessica I. Lake ${ }^{\dagger}$, Chara Malapani††, \\ and Warren H. Meck
}

The fidelity of timing and time perception in the milli-seconds to multiseconds range is subject to distortion in humans and other animals (DroitVolet and Meck, 2007; Eagleman, 2008; Matthews and Meck, 2014; Merchant et al., 2013a; Grondin, 2010). This failure to represent objective time in a veridical manner occurs in healthy individuals, including children, young adults, and aged participants as a function of temporal context (Balci et al., 20o9; Lustig and Meck, 2001, 2005, 2011; van Wassenhove et al., 2008). Of particular interest for this chapter, however, are the pathophysiological distortions in time perception and timed performance that occur as a function of dopamine (DA) depletion in the basal ganglia associated with Parkinson's disease (PD; see Allman and Meck, 2012; Coull et al., 2012, 2013; Harrington et al., 2014; Höhn et al., 2011; Jahanshahi et al., 2006; Jones et al., 2008; Jones and Jahanshahi, 2011, 2014). Patients with PD have a loss of DA neurons in the substantia nigra pars reticulata as well as the substantia nigra pars compacta (SNPC) which projects to the striatum - a brain region shown to be of critical importance to interval timing (Jahanshahi et al., 2006; Koch et al., 2008, 2009; Parker et al., 2013; Rakitin and Stern, 2006; Smith et al., 2007). PD patients experience difficulties when producing temporal components of movement, and in programming and synchronizing motor responses. These problems are reflected in increased reaction time and movement time (Bloxham et al., 1987; Evarts et al., 1981; Jurkowski and Stacy, 2005; Jurkowski et al., 2002, 2005a,b), prolonged

\footnotetext{
* Department of Psychology, University of Michigan, Ann Arbor, MI.

** Department of Psychology, University of Missouri, Columbia, Mo.

$\dagger$ Department of Psychology, University of California, Los Angeles, Los Angeles, CA.

†† Department of Psychiatry, Columbia University, New York, NY.

‡ Department of Psychology and Neuroscience, Duke University, Durham, NC.

(C) BON-MI GU ET AL., 2015 | DOI 10.1163/9789004230699_012

This is an open access chapter distributed under the terms of the Creative Commons

Attribution-Noncommercial 3,0 Unported (CC-BY=NC 3.0) License.
} 
inter-onset latencies when performing sequential or simultaneous movements (Benecke et al., 1986; Roy et al., 1993), impaired ability to maintain a fixed rhythm in tapping tasks (Nakamura et al., 1978; O'Boyle et al., 1996; Stelmach and Worringham, 1988; Wing et al., 1984) and increased speech production time (Lieberman et al., 1992; Volkmann et al., 1992). Parameters such as reaction time, inter-onset latency, rhythm maintenance, and temporal organization of speech all require accurate timing, and dysfunctions are well correlated with the clinical phenomenon of bradykinesia in PD (Benecke et al., 1986; Hallett and Khoshbin, 1980; Nakamura et al., 1978; Schirmer, 2004; Volkmann et al., 1992). There is evidence that the timing deficits in PD patients may not be limited to the motor domain. Non-motor deficits include impaired temporal discrimination of pairs of stimuli in the somaesthetic, visual, and auditory modalities (Artieda et al., 1992), absence of interlimb synchronization in processing of bimodal simultaneous stimuli (Malapani et al., 1994), difficulty in recognizing the emotional, linguistic, and temporal features expressed in speech (Schirmer, 2004), and impairments in attention, working memory, and time estimation (Brown and Marsden, 1988; Lee et al., 2010; Pastor et al., 1992; Perbal et al., 2005; Pouthas and Perbal, 2004). Recent evidence suggests that a unified model of timing and time perception may be possible based on coordinated activity in a core striatal network that is interconnected with olivocerebellar circuits and the cerebral cortex (Harrington et al., 2014; Koch et al., 2009; Kotz and Schwartze, 2011; Meck, 2005; Merchant et al., 2013a; Teki et al., 2012; see Gibbon et al., 1997, and Malapani et al., 1998a, for an alternative proposal for striato-thalamo-cerebral interconnectivity). This interactive timekeeping mechanism is used in both production and perception of intervals in the millisecond range and seconds-tominutes range with humans and other animals (Ivry and Hazeltine, 1995; Ivry and Keele, 1989; Jahanshahi et al., 2010a; Keele et al., 1985; Meck et al., 2008; Merchant et al., 2008b, 2013b). Thus, timing may be an independent/modular process employed whenever its specific computation is needed (Malapani and Gibbon, 2001; Merchant et al., 2013a; Van Rijn et al., 2014). In fact, accurate timing is required in many behaviors across species. For example, the skilled movements of musicians, athletes, and predators require precise timing of activity across different groups of muscles. It has been suggested that several motor or perceptual tasks that require accurate timing have access to the same internal timing mechanism, because normative performance varies in similar ways across tasks (Buhusi and Meck, 2005; Malapani and Fairhurst, 2002; Ivry and Hazeltine, 1995).

Neuropsychological research provides a complementary way to address the issue of whether a common timing mechanism is used in both production and perception and to investigate possible locations of the hypothesized internal timekeepers within neural systems (Coull et al., 2011; Meck et al., 2008; 
Merchant et al., 2013a). This approach asks whether different patient groups will show either similarities or dissociations in performance on specific timing tasks as a function of the location of their neurological lesion. It has been shown that patients with lesions in the cerebellum are impaired in both motor and perceptual tasks requiring accurate timing, in "short" sub-second time ranges (Ivry et al., 1988; Ivry and Keele, 1989) as well as in "long" suprasecond time ranges (Malapani et al., 1998b; Nichelli et al., 1996). Sub-second timing performance in perceptual and production timing tasks with comparable levels of difficulty was found to be unimpaired in PD patients (Ivry and Keele, 1989), leading to the hypothesis that timing in this range doesn't directly involve the basal ganglia (Ivry, 1996). However, more recent findings obtained in patients with diseases originating in the basal ganglia have shown that both time estimation and motor tasks requiring accurate timing are impaired in PD patients, in both sub-second and supra-second time ranges (Allman and Meck, 2012; Harrington et al., 1998a,b, 2011a,b; Jahanshahi et al., 2010a,b; Malapani et al., 1998b; Merchant et al., 2008a, 2013a; O'Boyle et al., 1996; Pastor et al., 1992).

\section{2 \\ Isolating the Internal Clock by Dividing Interval Timing into Separate Stages of Information Processing}

Distortions in timing that are induced by pharmacological, lesion, or stimulation techniques are postulated to be diagnostic of the underlying psychological processes (Allman et al., 2014a,b; Coull et al., 2011, 2013; Meck, 2006a,b,c). These temporal processes have been divided into three major stages of information processing (i.e., clock, memory, and decision stages - see Allman and Meck, 2012; Bueti and Macaluso, 2011; Hinton and Meck, 1997; Matell and Meck, 2000; Pouthas and Perbal, 2004) in order to isolate the underlying timing mechanisms (Roberts, 1981, 1993) and understand the time course of temporal discrimination (Tarantino et al., 2010).

\subsection{Clock Stage}

Systemic administration of DA agonists and antagonists has been found to cause horizontal leftward (e.g., amphetamine) and rightward (haloperidol) shifts in psychometric functions relating signal duration to temporal judgments (Lake and Meck, 2013; Maricq and Church, 1983; Meck, 1983, Meck et al., 2012a). These horizontal shifts are proportional to the durations being timed and have shown a positive correlation with the efficacy of the drugs at the D2 receptor (e.g., Meck, 1986). This result is compatible with the hypothesis that 
increasing (or decreasing) the effective level of DA in the brain causes an increase (or decrease) in the speed of oscillatory/pacemaker processes used for timing (Coull et al., 2011; MacDonald and Meck, 2004, 2005, 2006; Matell and Meck, 2004): if the clock is caused to run faster (or slower) than when a target duration was first learned, then participants will think that the end of the interval and its associated feedback is due earlier (or later) than it actually is, and consequently timing functions will be horizontally shifted to the left (or right). These horizontal shifts have been interpreted as indicative of effects upon "clock speed" (Cheng et al., 2006; Matell et al., 2004, 2006; Williamson et al., 2008). Astestingcontinuesundertheinfluenceofthedrug,however,theseunder-oroverestimations of signal duration begin to readjust such that by the end of testing, psychometric functions should have gradually shifted back to normal. If the drug is removed abruptly and testing continues, however, what one should see is an immediate rebound effect. That is to say, participants that have been tested with the DA agonists show a subsequent rightward shift upon removal of the DA agonist, while those tested with DA antagonists show a leftward shift. This is thought to be due to participants re-learning the target durations while under the influence of the drug (Buhusi, 2003; Meck, 1983, 1996). An example of how this might work is as follows: When participants have re-learned the target duration with a clock that runs faster (or slower) than normal, and are then returned to the "non-drug" baseline condition, the normal speed of the clock is slower (or faster) than it was during re-learning. Participants will then expect feedback later (or earlier) than the re-learned time, and the timing functions will be shifted to the right (or left), which is in the opposite direction to the shift induced by the initial administration of the drug (Oprisan and Buhusi, 2011).

\subsection{Memory Stage}

Horizontal displacement of psychometric functions may also result from distortions in the content of temporal memories that are stored for later retrieval (Coull et al., 2011; Meck, 1983, 1996). This distortion in the content of temporal memory has been described in terms of a multiplicative translation constant that is responsible for producing scalar transforms of sensory input taken from an internal clock (e.g., Meck, 2002a,b). An example of how this might work is as follows: the number of pacemaker pulses integrated by an accumulator during the presentation of a signal can provide a representation of the perceived duration for the current trial. When this clock reading is transferred to memory (presumably following feedback), the transfer may occur with some bias that we have referred to as a memory-storage or memory translation constant. In scalar timing theory (Gibbon et al., 1984; Wearden and Lejeune, 2008), this has 
formally been referred to as the $\mathrm{k}^{*}$ parameter, which is a multiplicative constant describing how the reference memory works during encoding and retrieval (Meck, 1983, 2002a; 2006c; Meck and Angell, 1992; Meck and Church, 1987; Meck et al., 1986; Van Rijn et al., 2014). If the remembered time of feedback reliably differed from the obtained clock reading, then a participant would consistently expect the feedback to occur at a time other than the programmed time. It would be later than the programmed time if the memory storage constant was greater than 1.o. Alternatively, the participant would consistently expect feedback to occur earlier than the programmed time if its memory storage constant was less than 1.0 (Meck, 1983, 2002a). A distortion at this level, known as the "memory-speed" effect, causes the real time of an event to be consistently remembered as "shorter" or "longer" than it actually is. Because the physical time is always misremembered, no amount of training will eliminate the distortion. Unlike changes in clock speed, changes in memory-storage speed would not be self-correcting even in the case where participants are "surprised" by the mismatch between their clock readings and a value sampled from reference memory. This is because any "updating" of memory in this case would lead to the continued distortion of stored values for as long as the modification in memory-storage speed remains in effect (i.e., as long as $\mathrm{K}^{*}$ is $>$ or $<1.0$ ). Pharmacological research has shown that the memory constant is related to the amount of acetylcholine (ACh) available in the brain, such that increasing (or decreasing) synaptic Ach decreases (or increases) the value of $K^{*}$ in a proportional manner to the timed interval (Meck, 1983, 1996, 2002a; Meck and Church, 1987; Oprisan and Buhusi, 2011).

\subsection{Decision Stage}

In virtually all interval-timing tasks, the decision to respond is based on the ratio of a currently accumulating duration to a remembered standard duration (Gibbon et al., 1984, 1997; Meck, 1983). If one observed decreasing variability with increasing target duration, the use of a difference rule rather than a ratio rule may be inferred, which would represent a violation of scalar variability across signal durations (Hinton and Rao, 2004; Rakitin et al., 1998; Wearden and Lejeune, 2008). Evidence regarding the neuropharmacology or anatomical locus of the decision stage is relatively lacking, but the interaction between the dorsal and ventral striatum has been implicated in the setting of response thresholds (Agostino et al., 2013; MacDonald et al., 2012). One piece of evidence concerning the neural processes that play a role in specific aspects of this decision process is the finding that participants systematically "search" for the target duration by systematically varying their temporal criterion on a trial-by-trial basis between a lower and upper boundary. This "temporal search" is revealed 
by an individual-trials analysis (Church et al., 1994; Rakitin et al., 1998) showing that successive peak times in the peak-interval procedure alternate between opposite sides of the median peak time more often that would be expected by chance (Meck, 1988; Meck et al., 1984). This finding suggests that duration discrimination mechanisms operate similar to a feedback-control system in order to minimize the amount of error in the estimation of the target duration and appear to depend upon hippocampal-striatal interactions (MacDonald et al., 2014; Meck, 1988; Meck et al., 2013; Yin and Meck, 2014; Yin and Troger, 2011). As such, a variety of feedback techniques have been used to affect this level of alternation and the decision stage in temporal generalization and reproduction tasks (e.g., Lustig and Meck, 2005; Wearden and Grindrod, 2003).

Recent work has demonstrated that human participants are sensitive to the probabilistic structure of the mean and variation of a distribution of event durations. This sensitivity can be modeled by a Bayesian-type decision process that participants use for the optimal estimation of duration and coincidence timing (Cicchini et al., 2012; Jazayeri and Shadlen, 2010; Miyazaki et al., 2005). These results highlight the statistical nature of psychophysical judgments as well as the efficiency and flexibility of decision-making processes involved in timing (Meck et al., 2012b). It has recently shown that the hyperbolic discounting of reward value emerges from the scalar property of interval timing (Cui, 2011). Moreover, there is an increasing awareness that decision-making is influenced by subjective time being reliably different from objective time and that these differences are modulated by sources and forms of variability in clock, memory, and decision stages (Gibbon et al., 1997; Gu and Meck, 2011; Meck et al., 2012b; Jazayeri and Shadlen, 2010; Ray and Bossaerts, 2011).

In summary, there are a number of important implications derived from the findings described above: (1) clock and memory stages of temporal processing are functionally distinguishable; (2) DA function appears to be importantly involved in the "clock" stage and perhaps in the "memory" stage as well although Ach function appears to be more strongly implicated (Coull et al., 2011; Oprisan and Buhusi, 2011). However, findings in bilateral PD patients with substantial losses of DA cell bodies in the SNPC, show reliable "migration effects" when trained and tested with multiple target durations during the same session. This outcome was unanticipated based on the hypothesis that DA is solely involved in the clock stage - which would predict a proportional rightward shift for both target durations as clock speed slows down rather than migration towards each other (Malapani et al., 1998b; Meck and Benson, 2002). These PD findings also challenge current timing theories (e.g., scalar timing theory and striatal beat-frequency theory, SBF; Gibbon et al., 1984, 1997; Matell 
and Meck, 2004), which cannot account for all of the quantitative properties of the observed clock and memory effects without some additional assumptions about memory processes and decision-making (Gu and Meck, 2011; Lustig et al., 2005). We describe these effects as part of the review below.

\section{Migration Effects Observed in Temporal Reproduction}

The peak-interval (PI) timing procedure is a duration reproduction task in which human participants are instructed to reproduce durations by centering a sequence of responses around the target duration(s) that typically range from 6-21 s (Rakitin et al., 1998; Yin et al., 2015). The target duration is initially pre-trained by presenting the participant with a series of fixed-time (FT) trials (e.g., $\mathrm{N}=10$ trials) during which the signal (e.g., blue rectangle) briefly changes color (blue-> magenta) at the target duration. Participants were then asked to reproduce this duration from memory during peak trials by either centering a sequence of key presses (e.g., Lake and Meck, 2013; Lustig and Meck, 2005; Malapani et al., 1998b; Rakitin et al., 1998, 2005, 2006) or pressing a key just before and releasing it just after the target duration (Malapani et al., 2002) before terminating the trial and the continuous presentation of the blue rectangle. Trials are separated by an intertrial interval (ITI) during which a feedback histogram can be presented on the computer screen displaying the frequency of responses plotted on a relative time scale with the letter " $\mathrm{T}$ " marking the target duration and a bracket of $\pm 15 \%$ displayed around this target duration - thus, providing information concerning the accuracy and precision of the responses produced on the immediately preceding trial. Additional messages informed the participants if they had responded too short or too long with respect to the target duration or whether one of their responses was a direct hit. Participants were instructed that they should refrain from counting or tapping so as to avoid sub-dividing or any other type of extraneous timekeeping. To further discourage counting, random digits were intermittently superimposed over the blue rectangle, and participants were instructed to read the digits aloud. Inter-digit times were determined randomly using two uniform distributions (see Malapani et al., 1998b; Rakitin et al., 1998, for additional details). In the following sessions, participants typically received $20 \mathrm{FT}$ trials, 30 peak trials with ITI feedback, and 10 peak trials without ITI feedback during blocked sessions each with a different target duration over the course of two or more days. Data collection sessions were preceded by pre-training sessions during both days. Target duration pairs used were: 11 and $17 \mathrm{~s}$ (Hinton, 2003; Hinton and Meck, 2004; Meck and Malapani, 2004), 7 and $17 \mathrm{~s}$ (Lake and 
Meck, 2013), 7 and $14 \mathrm{~s}$ (Gu et al., 2015; Lustig and Meck, 2005), 8 and $21 \mathrm{~s}$ (Malapani et al., 1998b), and 6 and $17 \mathrm{~s}$ (Malapani et al., 2002; Rakitin and Malapani, 2005; Rakitin et al., 2005, 2006).

Malapani et al. (1998b) showed that when PD patients given DA-replacement therapy (e.g., L-dopa + apomorphine) are trained to time using corrective feedback they are able to reproduce single or multiple target durations (e.g., 8 and/ or $21 \mathrm{~s}$ visual signals trained individually or within the same session) with the same accuracy and precision as age-matched controls. However, when these same PD patients are tested in an OFF medication state (again with feedback) a very different phenomenon emerges. Under these conditions, the $8 \mathrm{~s}$ target duration is over-produced and the 21s target duration is under-produced relative to the ON medication state when both durations are trained and tested within the same session, as illustrated in Figure 11.1. This distortion was characterized as a "migration" effect, as though there was a mutual attraction, or "coupling" in memory for these two target durations. It is important to note that the $21 \mathrm{~s}$ target duration was reproduced as "shorter" than veridical when there was a resident memory of an $8 \mathrm{~s}$ target duration trained previously, but was reproduced as "longer" than veridical when it was the only trained target duration tested in the OFF medication state, indicating a "slowing" of temporal processing (e.g., Fournet et al., 2000; Meck and Benson, 2002) as illustrated in Figure 11.2.

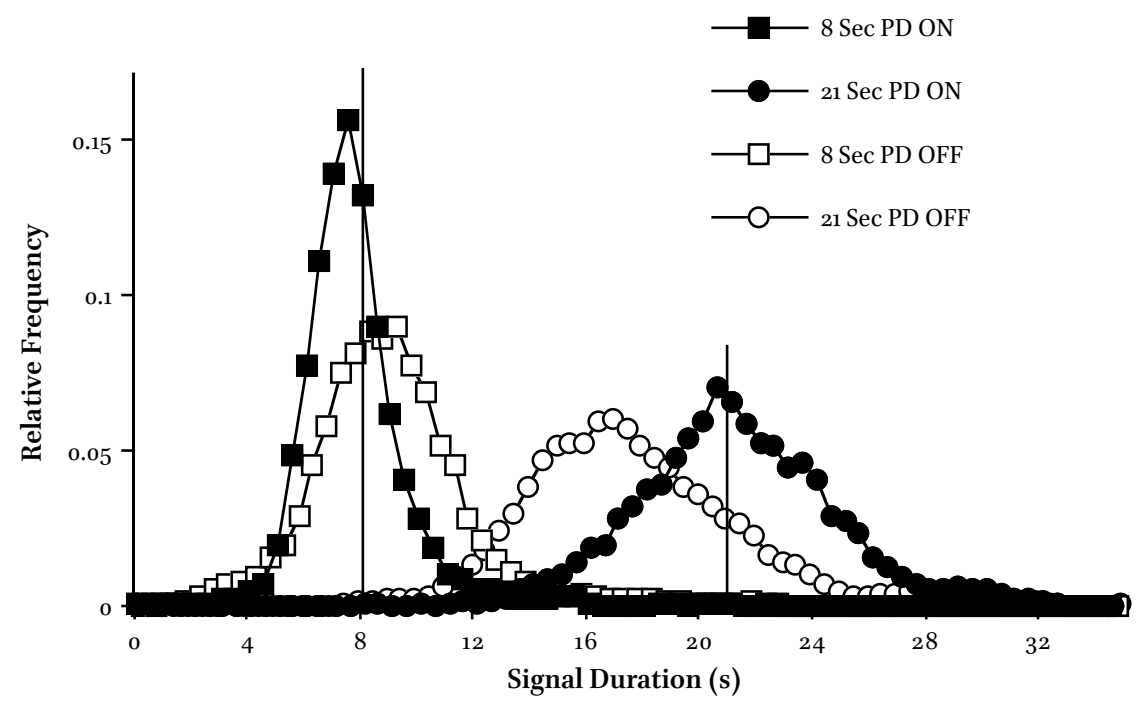

FIGURE 11.1 Relative frequency distributions for the two target durations ( 8 and $21 \mathrm{~s}$ ) showing accuracy and precision of duration reproduction in Parkinson's disease (PD) patients ON and OFF their levodopa medication. 


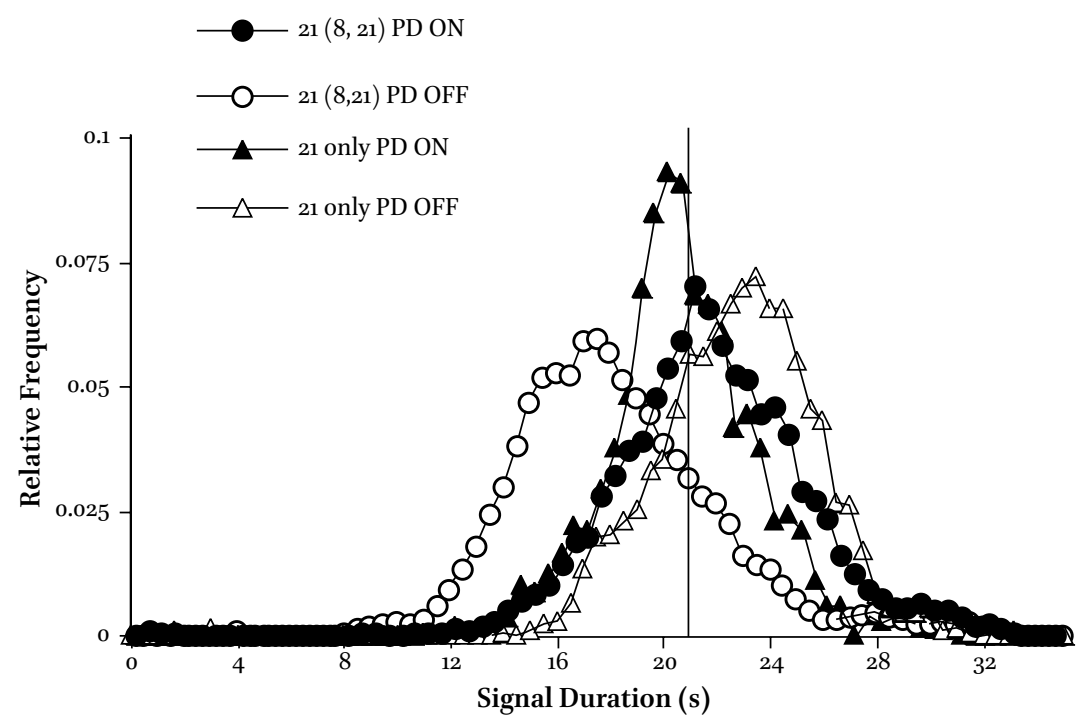

FIGURE 11.2 Relative frequency distributions for a 21-s target duration plotted as a function of whether it was trained in conjunction with a 8-s target duration or by itself (21 only). The peak functions illustrate the accuracy and precision of duration reproduction in Parkinson's disease (PD) patients ON and OFF their dopaminergic medication as a function of the multiple or single target duration training.

These findings have been followed up using an "encode/decode" paradigm (Malapani et al., 2002) that is able to reveal whether the dysfunctional coupling is part of the original learning and storage of the appropriate target durations (encoding) or introduced upon retrieval of these memories at test (decoding). Using this training (with feedback) $\rightarrow$ test (without feedback) paradigm, it was demonstrated that the migration effect appears whenever PD patients are tested OFF medication, whether this is during training or test phases of the experiment. These results replicate and extend the earlier PD-migration effect first reported by Malapani et al. (1998b) showing that compared to the ON medication state, $\mathrm{PD}$ patients tested OFF medication produce responses that are "long" relative to the "short" target duration and responses that are "short" relative to the "long" target duration. The PD-migration effects reported by Malapani et al. (2002) were observed during both of the OFF training sessions, as seen in the OFF-OFF and OFF-ON groups' first-day performances, and in the test sessions, as seen in the OFF-OFF and ON-OFF groups' second-day performances. These results indicate that the PD-migration effect is not entirely dependent on the presence or absence of ITI feedback and is unlikely attributable to a selective problem with memory encoding. These findings led Malapani et al. (2002) to attribute migration in PD patients to a 
DA-dependent dysfunction of retrieving temporal memories. A different type of timing deficit was observed in the OFF-ON groups' data from the test session. In this case, PD patients substantially overproduced both target durations. These data from the OFF-ON group contrast with the test session data from the OFF-OFF group, which clearly show strong migration. Apparently, restoring DA function during the test session improves the retrieval deficit (manifested as migration) and allows a second, clock slowing/encoding deficit associated with DA deficiency during the training session to be observed in a manner similar to what has been reported for adult attention-deficit disorder patients tested off of their dopaminergic therapy (Allman and Meck, 2012; Meck, 2003, 2005) or patients given chronic treatment with haloperidol, a DA receptor blocker (Lustig and Meck, 2005).

In addition to the magnitude and direction of errors in accuracy (e.g., leftward or rightward horizontal shifts in peak time - see Lustig and Meck, 2005; Malapani et al., 1998b; Meck, 2002a,b; Meck and Benson, 2002), DA-dependent distortions in storage and retrieval are also distinguishable by changes in the source and pattern of variability in treated and untreated PD patients (see Jones et al., 2011). Analysis of timing data precision showed that the PDmigration effects were associated with a violation of the scalar property of interval timing (Gibbon et al., 1997; Hinton and Rao, 2004; Rakitin et al., 1998). That is, the relative widths of the Gaussian-shaped PI response distributions for the two target durations were systematically different from each other when responses are plotted in proportion to the maximal level of responding on a relative time scale - such that the $21 \mathrm{~s}$ function was sharper than the $8 \mathrm{~s}$ function as illustrated in Figure 11.3. Timing functions for multiple target durations would normally be expected to superimpose when plotted on a relative time scale as a result of scalar sources of variance dominating non-scalar (e.g., Possion) sources (see Cheng and Meck, 2007; Gibbon, 1992; Rakitin et al., 1998). Moreover, the coefficient of variation (CV) expressed as the standard deviation of timed responses expressed as a proportion of the obtained peak time should be constant (Gibbon et al., 1997), but was observed to be different for the two target durations in all PD groups tested OFF medication, replicating the previous findings of Malapani et al. (1998b). The storage deficit associated with the rightward shifts for both target durations, however, continued to reflect the scalar property of interval timing; that is, the standard deviation of response times increased in proportion to the magnitude of the timing errors (Malapani et al., 2002).

In summary, DA deficiency leading to dysfunction in the basal ganglia results in two separable temporal memory deficits (see Malapani and Rakitin, 2003; Shea-Brown et al., 2006). Distortions that occur while storing multiple 


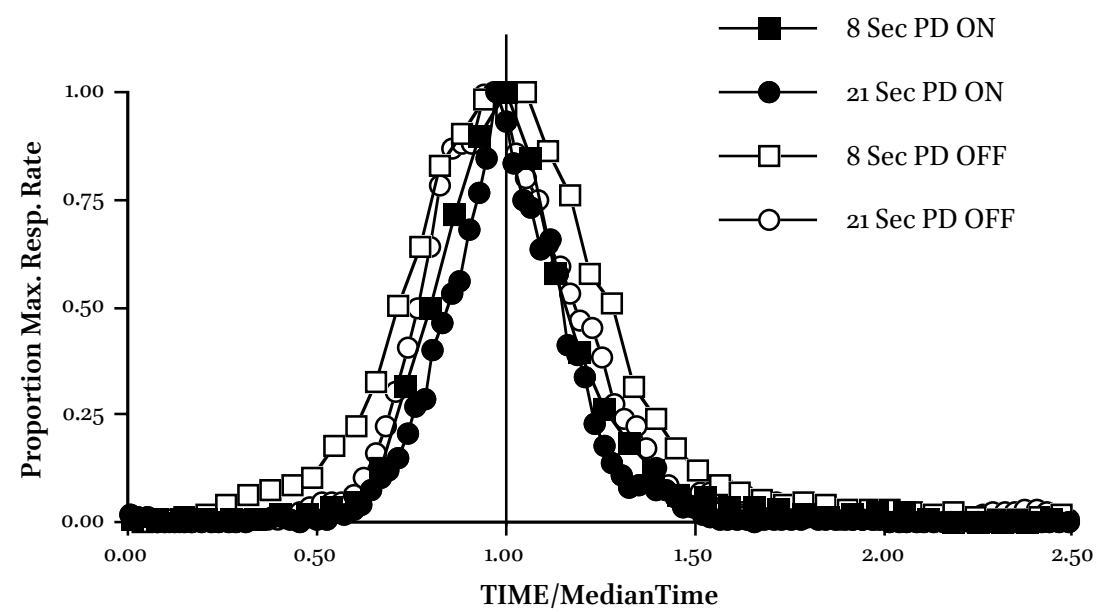

FIGURE 11.3 Proportion maximum response rate for 8- and 21-s target durations for Parkinson's disease (PD) patients trained ON and OFF their levodopa medication. Data are plotted in time relative to the median time and normalized as proportions of the maximum response rate/frequency. Peak functions superimpose in the ON medication state, but not in the OFF medication state - indicating a violation of the scalar property of interval timing with lowered dopaminergic function.

target durations result in overestimation of all intervals during subsequent reproduction. In contrast, retrieving the trace of two or more different target durations results in migration, a pattern of bidirectional errors such that reproductions of one of a pair of target durations are unusually close to the reproductions of the second. In addition to migration, DA-deficient retrieval is accompanied by a violation of the scalar property of interval timing, whereas with unidirectional shifts, the scalar property holds. It is important to note here that estimation of continuous attributes other than time (e.g., line length) is spared in PD in an analogous "encode/decode" paradigm (Malapani and Fairhurst, 2002). Moreover, patients with other kinds of brain damage, such as focal cerebellar lesions, do not show the temporal distortions seen in PD (Ivry, 1996; Malapani et al., 1998b, 2002). Taken together, this evidence strongly suggests that the basal ganglia and their cortical targets are an important aspect of the neural basis of interval timing in humans, and seem to involve both a slowing of "clock speed" and a "memory migration" component when PD patients are withdrawn from their dopaminergic medication (Buhusi and Meck, 2005; Fournet et al., 2000; Meck and Benson, 2002).

Migration in temporal memory has also been reported in healthy aged participants, when no further feedback or training is available. Although migration in normal aging appears similar to the migration observed in PD patients 
when tested OFF medication, there are distinguishable features between the effects attributable to normal age-related decline and those observed in PD patients (Lustig, 2003; Rakitin and Malapani, 2008; Wojtecki et al., 2011). First, the absolute size of the migration effect is usually smaller in healthy older participants than in older PD patients. Second, feedback typically eliminates the migration effect observed in older healthy individuals, but not in older PD patients. Third, the relation between the duration of the interval reproduced and the variability of that production more closely reflects Weber's law for healthy older participants than for older PD patients. These differences suggest that at a minimum, the age-related deficit is less severe than the PD-related deficit, and can be alleviated with the presentation of feedback. On the other hand, the differences may imply an additional mechanism at play in PD that obviates the usefulness of feedback and adds a non-temporal related variability component (Rakitin and Malapani, 2008; Rakitin et al., 2005). Yet another difference between the two groups was that all PD patients tested OFF medication show migration, whereas the effect is considerably more variable among healthy aged participants. Aged participants, for example, can be divided into sub-groups that show: (a) rightward shifts for both durations, (b) migration towards the mean for both durations, or (c) non-systematic shifts for both durations consistent with random variation in veridical timekeeping (Rakitin et al., 2005). These data raise the possibility that the observation of the "migration effect" in apparently healthy older adults may be an early, sensitive measure of age-associated cognitive decline, further suggesting that interval-timing paradigms may be an important tool for early detection of PD (Jurkowski and Meck, 2003). This hypothesis can be further tested by administering aged participants levodopa or placebo treatments as a function of training and test sessions in the PI and other timing procedures (Rakitin et al., 2006).

The neural basis of the PD-migration effect and the other temporal-processing deficits exhibited by PD patients are still poorly understood. The "migration effect" has been reported to be more evident in left hemi-Parkinsonian patients (Koch et al., 2005) - a finding that supports the lateralization of interval timing to the right hemisphere (Ferrandez et al., 2003; Harrington et al., 1998a,b; Meck, 2005; Meck and Malapani, 2004; van Wassenhove et al., 2008). In addition to dopaminergic medication (e.g., L-dopa), timing deficits in PD are alleviated by repetitive transcranial magnetic stimulation (rTMS) of the right dorsolateral prefrontal cortex (DLPFC), suggesting that impaired evidence-accumulation and decision-making processes contribute to distortions in timing and time perception (Allman and Meck, 2012; Jones et al., 2011; Kim and Lee, 2011; Koch et al., 2003, 2004; Philiastides et al., 2011; Rahnev et al., 2011). Traditional accounts of timing and time perception have used a pacemaker-accumulator 
TABLE 11.1 Temporal migration effects in Parkinson's disease.

\begin{tabular}{llll}
\hline $\begin{array}{l}\text { PD migration } \\
\text { studies }\end{array}$ & $\begin{array}{l}\text { Reproduction } \\
\text { durations }\end{array}$ & $\begin{array}{l}\text { Train-Test DA } \\
\text { replacement } \\
\text { therapy conditions }\end{array}$ & Feedback conditions/notes \\
& & \\
\hline
\end{tabular}

\begin{tabular}{|c|c|c|c|}
\hline Dušek et al. (2012) & $\begin{array}{l}5,5.95,7.07 \\
8.41,10,11.89 \text {, } \\
14.14 \text {, and } \\
16.81 \mathrm{~s}\end{array}$ & ON-OFF & No feedback \\
\hline Gu et al. (2015) & $7 \mathrm{~s}$ and $14 \mathrm{~s}$ & ON-OFF & $\begin{array}{l}25,50 \text {, and } 100 \% \text { peak } \\
\text { trials with IT I feedback }\end{array}$ \\
\hline Gu et al., (2015) & $\begin{array}{l}7,7.875,9.625 \\
10.5,11.375 \\
12.24,13.125 \\
\text { and } 14 \mathrm{~s}\end{array}$ & ON-OFF & No feedback \\
\hline Koch et al. (2004) & 5 and $15 \mathrm{~s}$ & ON-OFF & $\begin{array}{l}\text { No feedback - DBS and } \\
\text { DA therapy }\end{array}$ \\
\hline Koch et al. $(2005)$ & 5 and $15 \mathrm{~s}$ & ON-OFF & $\begin{array}{l}\text { No feedback - left and } \\
\text { right hemi-PD* }\end{array}$ \\
\hline Koch et al. (2008) & $500 \mathrm{~ms}$ and $2 \mathrm{~s}$ & ON-OFF & No feedback** \\
\hline $\begin{array}{l}\text { Malapani et al. } \\
(1998 b)\end{array}$ & $\begin{array}{l}8 \text { and } 21 \mathrm{~s}, 21 \mathrm{~s} \\
\text { alone }\end{array}$ & ON-OFF & $\begin{array}{l}\text { FT trials }+66 \% \text { peak } \\
\text { trials with IT I feedback } \\
\text { during both ON and } \\
\text { OFF }\end{array}$ \\
\hline $\begin{array}{l}\text { Malapani et al., } \\
(2002)\end{array}$ & 6 and $17 \mathrm{~s}$ & $\begin{array}{l}\text { ON-ON; ON-OFF; } \\
\text { OFF-ON; } \\
\text { OFF-OFF }\end{array}$ & $\begin{array}{l}\text { FT trials }+75 \% \text { peak } \\
\text { trials with ITI feedback } \\
\text { during ON, no feedback } \\
\text { during OFF }\end{array}$ \\
\hline $\begin{array}{l}\text { Wojtecki et al. } \\
(2012)\end{array}$ & 5 and $15 \mathrm{~s}$ & $\begin{array}{l}\text { Controls vs PD } \\
\text { OFF }\end{array}$ & $\begin{array}{l}\text { No feedback - DBS } \\
\text { frequency varied }\end{array}$ \\
\hline
\end{tabular}

DA = dopamine; $\mathrm{DBS}=$ deep brain stimulation; $\mathrm{FT}$ = fixed-time; $\mathrm{ITI}$ = intertrial interval; $\mathrm{PD}=$ Parkinson's disease

* Only left hemi-PD patients showed the full PD-migration effect. Right hemi-PD patients showed an overproduction for the "short" (5 s) duration (Koch et al., 2005).

** Failed to see the full PD-migration effect when the "short" duration was in the milliseconds range (50oms) and the "long" duration was in the seconds range $(2 \mathrm{~s})$, although the 2-s duration did shift leftward (Koch et al., 2008). 
model to explain how the internal clock might function (Gibbon et al., 1984; Shea-Brown et al., 2006; Treisman, 1963). These models have been replaced by more physiologically-realistic theories that rely on the coincidence-detection of cortical oscillations by striatal medium spiny neurons - referred to as the SBF model of interval timing (Agostino et al., 2011; Allman and Meck, 2012; Buhusi and Oprisan, 2013; Matell and Meck, 2004; Matell et al., 2003; Oprisan and Buhusi, 2011, 2013). According to the SBF model, thalamo-cortico-striatal circuits learn to detect specific durations by associating feedback with particular patterns of cortical oscillations. Interestingly, pathological alterations of neuronal oscillations have recently been implicated in the pathophysiology of PD symptoms (Brown, 2003; Brown et al., 2001; Eusebio et al., 2012; Hutchison et al., 2004; Timmermann et al., 2003, 2004). Importantly, a number of recent studies have demonstrated that timing deficits in PD are alleviated by deep brain stimulation of the subthalamic nucleus (Koch et al., 2004) with differential effects of stimulation frequency (Wojtecki et al., 2011). These findings support the importance of specific oscillatory processes in that stimulation of the subthalamic nucleus differentially modulates motor and timing functions depending on stimulation frequency (Timmermann et al., 2004; Wojtecki et al., 2006). A summary of the PD-migration effects described above is provided in Table 11.1.

\section{4. $\quad$ Effects of Corrective Feedback on Interval Timing}

In PI timing experiments there are generally two types of feedback given, FT trials that demonstrate the target duration and ITI histograms that inform participants of the relative accuracy and precision of responses made during the preceding peak trial (Malapani et al., 1998b, 2002; Lake and Meck, 2013; Lustig and Meck, 2005; Rakitin and Malapani, 2008; Rakitin et al., 1998, 2005). It can, thus, be postulated that corrective feedback has two components: (1) a memory component in which participants use the FT trial color change to update their memory of the target duration and (2) a threshold-setting component in which participants use the ITI histogram for purposes of correcting errors in start and stop times (see Church et al., 1994; MacDonald et al., 2012). Although one could argue that FT trials might also affect threshold setting, the findings from a number of experiments suggest that these two types of feedback have distinct effects on timing behavior (Lustig and Meck, 2005; Rakitin and Malapani, 2008; Rakitin et al., 1998).

Lustig and Meck (2005) investigated the effects of ITI feedback in the PI procedure (7- and 14-s target durations) as a function of the probability of feedback $(25,50$, or $100 \%)$ and the blockade of DA receptors by haloperidol. Within 
the $25 \%$ feedback condition the effect on peak time of the number of "trials since feedback" - which reflects the decay of feedback effects over time - was also investigated. The results showed an interaction between the feedback and drug conditions such that a proportional over-production of peak time was observed for the 7- and 14-s target durations under chronic haloperidol that increased as the probability of feedback was reduced as illustrated in Figure 11.4. Moreover, a trial-by-trial analysis demonstrated that reproduction of the target interval becomes later in time as feedback becomes more remote. This effect occurs for both control and drug treatment, but was exaggerated under haloperidol. This pattern of results raises some interesting questions about the mechanisms by which feedback has its effects, and the interactions of those effects with dopaminergic function. Feedback is often thought to have its impact by updating the representation of the target duration in memory (see discussions by Matell and Meck, 2004; Meck, 1996; Meck and Benson, 2001). That is, feedback serves to alert the participant that the amount of time they have associated with the target duration is incorrect, and on subsequent trials they should change the amount of time before responding in order to adjust for the discrepancy (Meck, 2002a,b). For example, in the current experiment, where DA receptor blockage and distance from feedback led to an overproduction of time, the ITI feedback would alert participants that they were waiting too long and that on the next trial they should respond earlier. Consequently, one possible explanation for the pattern of results found here is that the representation of the target duration and/or the corrective effects of feedback in working memory decay over time, and that this decay is exaggerated by the blockade of DA receptors. This interpretation would be consistent with previous findings that chronic haloperidol administration downregulates the D1 sub-type of DA receptors and impairs working memory (Castner et al., 2000), and that DA release is important for the correction effects of feedback observed in many tasks (e.g., Schultz, 1998; Schultz and Dickinson, 2000 but see Kim et al., 2014).

Another possibility is that both feedback and haloperidol had direct effects on the clock stage given that DA antagonists such as haloperidol have been shown to decrease "global" clock speed during the timing of all trials in the PI procedure (e.g., Buhusi and Meck, 2002; MacDonald and Meck, 2005, 2006; Meck, 1986; Lake and Meck, 2013; Williamson et al., 2008). As a consequence, the dynamic and proportional rightward shifts observed as a function of the number of "trials since feedback" can be viewed as "local" effects on clock speed if one assumes that the clock slows down as the DA-releasing effects of feedback wear off and is temporarily speeded up again when feedback is delivered (e.g., Allman and Meck, 2012; Kawashima et al., 2012; Koepp et al., 1998; Matell and Meck, 2004). This proposal doesn't distinguish between positive 


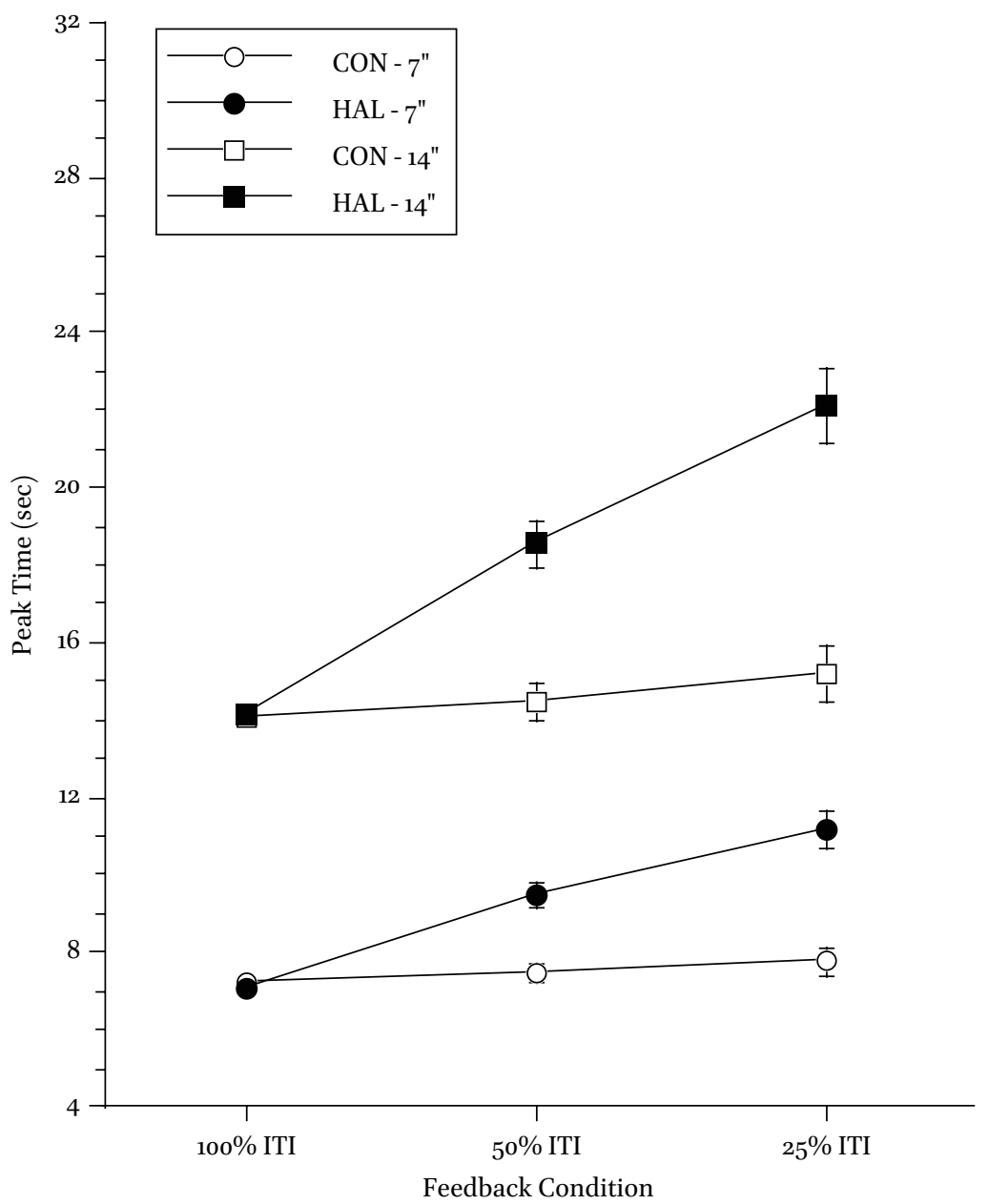

FIGURE 11.4 Peak times (means $\pm S E$ ) for participants in the control (CON) and haloperidol (HAL) conditions tested with 7-and 14-s target durations in the peak-interval procedure. Data are plotted as a function of the probability of intertrial interval (ITI) feedback $(25,50$, and $100 \%)$.

and negative feedback and assumes that the information provided by the ITI feedback is equally "stimulating" (at least in healthy participants) regardless of whether it indicates that they are doing well or have made an error. It is, of course, possible to separate these effects under conditions where participants make numerous errors (i.e., do not place their responses within the bracketed window displayed in the ITI histogram), but in practice young control participants do not make many errors of this sort even under the $25 \%$ feedback 
condition. It's interesting to note that when aged participants are tested without feedback following an initial training session with feedback, there is a migration effect regardless of the type and amount of feedback given during training. Moreover, it was observed that false and inconsistent feedback induces migration in the elderly of an order of magnitude similar to the pdmigration effect (Rakitin and Malapani, 2008; Rakitin et al., 2005).

Importantly, PD patients evaluated $\mathrm{ON}$ and OFF their dopaminergic medication using the same ITI feedback probabilities $(25,50$, and $100 \%)$ and target durations ( 7 and $14 \mathrm{~s}$ ) as Lustig and Meck (2005) showed effects different from young participants evaluated under haloperidol. When age-matched controls were trained with 7- and 14-s target durations, they showed a tendency to shift rightward as ITI feedback became more infrequent. In contrast, PD patients in both the ON and OFF medication states showed a "migration effect" that increased as ITI feedback became more infrequent such that the 7-s target duration was over-produced and the 14-s target duration was under-produced. This "migration effect" however, was substantially larger in the PD-OFF medication state than in the PD-ON medication state (in which significant migration was only observed for the 25 and 50\% ITI conditions) as illustrated in Figure 11.5. Relating these effects to clock speed, memory encoding/decoding, and error processing presents an interesting challenge to understanding the cognitive deficits associated with PD and DA depletion (see Nagano-Saito Atsuko et al. 2008).

\section{Mismatch Theory and the Error-Related Negativity}

Error processing reflects the brain's ability to detect and adjust to incorrect behaviors (e.g., Falkenstein et al., 200o). Initially, investigators hypothesized that error processing involved a comparison between a participant's actual response and the representation of the correct response stored in memory (e.g., Nieuwenhuis et al., 2002). Studies investigating this "mismatch theory" found an electrophysiological component of error processing, the error-related negativity (ERN; Falkenstein et al., 200o; Herrmann et al., 2004; Holroyd and Coles, 2002; Nieuwenhuis et al., 2002). The ERN is a negative shift in the recorded electroencephalogram (EEG) believed to be the unconscious aspect of error processing and appears approximately 8oms following an error (Holroyd and Coles, 2002). The ERN is typically measured at the peak height of the negative deflection.

According to the initial mismatch theory of error processing, the ERN follows an error in order to represent the error as a difference between the actual response and the correct response. For correct responses, little if any 


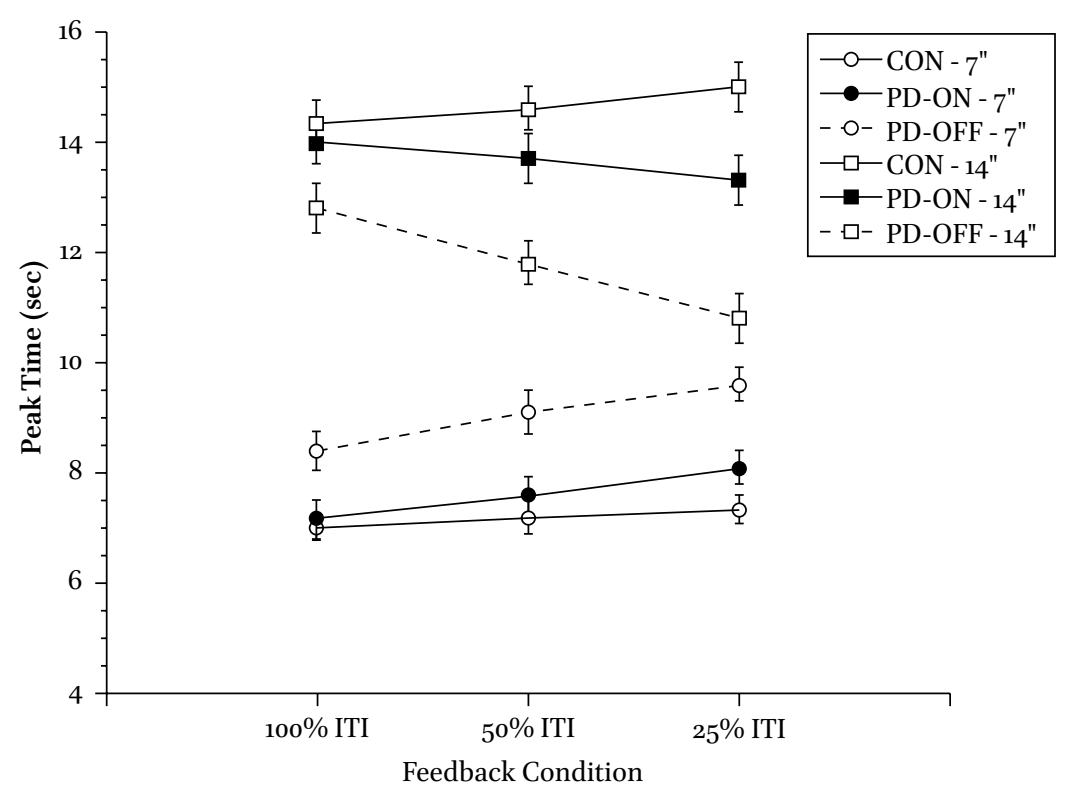

FIGURE 11.5 Peak times (means $\pm S E$ ) for Parkinson's disease (PD) patients trained ON and OFF their dopaminergic medication and aged-matched controls using 7 - and 14-s target durations in the peak-interval procedure. Data are plotted as a function of the probability of intertrial interval (ITI) feedback (25, 50, and $100 \%)$.

difference should exist between the actual response and the correct response. Thus, the ERN should appear following errors only, but not correct responses. However, experimental evidence has shown that a comparatively less negative ERN followed correct responses in addition to the ERN that followed errors (Herrmann et al., 2004). The ERN following correct responses refuted the initial mismatch theory, and a refined version of the theory was developed to explain the presence of the $\mathrm{ERN}$ following both correct and incorrect responses. The refined mismatch theory combines the ERN with a reinforcement-learning algorithm referred to as the "method of temporal differences," which relies upon the mesencephalic DA system for updating (Holroyd and Coles, 2002). According to the refined mismatch theory, the ERN should occur when the mesencephalic DA system produces a negative reinforcement signal (less DA release following an error than during baseline) to the anterior cingulate cortex (ACC), the region of the brain believed to generate the ERN (Nieuwenhuis et al., 2002; San Martín, 2012). This negative reinforcement signal represents the DA system's response to negative feedback when an error is made. Negative feedback occurs when the participant is notified that he/she made an error; 
however, even in the absence of explicit information, it is possible that the DA system can still transmit negative feedback following the error. Specifically, in a task that involves judging different signal durations, for example, the range and other statistics defining the distribution of signal durations might become stored in the participant's reference memory and, thus, provide internal feedback. This type of feedback is also thought to depend on the levels of DA in cortico-striatal circuits (Buhusi and Meck, 2005; Cools et al., 2002; de Vries et al., 2010; Nieuwenhuis et al., 2004; Wise, 2004).

\subsection{Dopamine Effects on the Error-Related Negativity}

After an error occurs, the ACC detects the extent to which DA levels decrease, and accordingly, the amplitude of the ERN depends on how much the actual response differs from the correct response (Holroyd and Coles, 2002). The ERN amplitude for correct responses reflects the smallest possible difference between the actual response and the correct response, and the ERN of an error is expected to have a more-negative amplitude than the ERN of a correct response. The refined mismatch theory thereby accounts for the less negative ERN found after correct responses by proposing that the ERN amplitude is regulated by the amount of DA released in specific pathways. The amount of DA released by the mesocortical and nigrostriatal pathways also depends on biological factors. Natural degeneration of the mesocortical and nigrostriatal pathways occurs as healthy adults age (Fearnley and Lees, 1991; Rubin, 1999) and severe degeneration of the mesocortical and nigrostriatal pathways occurs as a result of $\mathrm{PD}$. Thus, the presumed reduction in $\mathrm{DA}$ in these groups should result in a lowered response to negative feedback. To test these predictions concerning the effects of DA level on error processing, past studies of the ERN compared healthy young adults to healthy aged adults (Kolev et al., 2005) or healthy aged adults to PD patients (Holroyd et al., 2002; Ito and Kitagawa, 2006) by comparing correct responses with errors and by comparing the ERN amplitude for errors across groups.

\subsection{Age Effects on the Error-Related Negativity}

Kolev et al. (2005) used a choice reaction time task to examine differences in error processing due to age and found that the ERN amplitude was less negative in correct responses compared to incorrect responses for both young and aged adults, as consistent with studies of the initial mismatch theory (Herrmann et al., 2004). In a comparison between groups, however, the ERN amplitude was more negative in young adults compared to aged adults. These results were interpreted as support for the refined mismatch theory because age is expected to decrease DA levels. The more negative ERN amplitude in 
young adults was also interpreted to indicate that error detection is impaired by age (Kolev et al., 2005).

\subsection{Parkinson's Disease Effects on the Error-Related Negativity}

Holroyd et al. (2002) tested aged adults and PD patients OFF dopaminergic medication using a choice reaction time task and found that the ERN amplitude was similar across aged adults and PD patients. The ERN amplitude of correct responses was not directly compared to incorrect responses within each group. Instead, the ERN of correct responses was subtracted from the ERN of incorrect responses for comparisons between groups. Because the ERN was still present after the subtraction, the ERN amplitude of correct responses was assumed to be less negative than the ERN amplitude of incorrect responses. The similar ERN amplitude across aged adults and PD patients tested OFF medication was interpreted to indicate that PD did not reliably impair error detection (Holroyd et al., 2002). In contrast, Ito and Kitagawa (2006) tested aged adults and PD patients ON dopaminergic medication using a lexical decision task and found that the ERN amplitude was less negative in correct responses compared to incorrect responses for both aged adults and PD patients, as consistent with studies of the initial mismatch theory. Between groups, the ERN amplitude was more negative in aged adults compared to PD patients. These results were interpreted to support the refined mismatch theory even though dopaminergic medication is thought to increase DA levels in PD patients and make them more similar to age-matched controls. The more negative ERN amplitude in aged adults was also interpreted to show that PD impaired error detection (Ito and Kitagawa, 2006). As a consequence, it is still an open question as to the relationship between error processing, DA levels, and the ON and OFF medication states of PD.

\section{Affective-processing Hypothesis of the Error-Related Positivity}

The error positivity $(\mathrm{Pe})$ is a positive deflection in the EEG believed to be the conscious component of error processing (Overbeek et al., 2005). The Pe appears approximately $200-500 \mathrm{~ms}$ following an error and is measured at the peak height of the positive deflection (Falkenstein et al., 2000, 2005). According to the affective processing hypothesis of error processing, the Pe reflects post-error processing in the form of subjective/emotional assessment (Falkenstein et al., 200o) and is independent of the ERN (Overbeek et al., 2005). The amplitude of the Pe is believed to indicate the emotional 
significance of an error (Falkenstein et al., 2000). A study of the affective processing hypothesis compared participants who made numerous errors with participants who made relatively few errors and found that the amplitude of the Pe was more positive in participants who made few errors compared to participants who made many errors. The results were interpreted to support the affective processing hypothesis because errors were assumed to have less emotional significance for participants who made many errors compared to participants who made few errors - although the Pe has not been investigated following correct responses (Falkenstein et al., 2000).

The Pe follows the ERN in time, but is considered independent of the ERN because the Pe and ERN are generated by different areas of the ACC. The ERN appears in the medial prefrontal region of the ACC while the Pe appears in a more rostral region of the ACC (Herrmann et al., 2004). Also, unlike the ERN, the Pe is not present in all errors. For example, Ehlis et al. (2005) tested the affective processing hypothesis using a negative response feedback task, in which participants were sometimes told that they responded incorrectly when they actually responded correctly (false feedback). This study found that the Pe was present following incorrect responses but was not present following negative response feedback, possibly because participants expressed certainty that they had responded correctly. The assumed independence of the Pe from the ERN is also supported by evidence that the DA system does not appear to affect the Pe. Falkenstein et al. (2005) found that the Pe was similar in healthy aged adults compared to PD patients evaluated OFF dopaminergic medication even though PD reduces DA levels beyond the effects of normal aging. Moreover, Ito and Kitagawa (2006) found that the Pe amplitude was more positive in healthy aged adults compared to PD patients tested ON dopaminergic medication, even though medication is thought to increase DA levels in relevant brain circuits in PD patients. Although the ERN and Pe have been studied in lexical decision (Kolev et al., 2005), choice reaction time (Ito and Kitagawa, 2006), and negative feedback tasks (Ehlis et al., 2005), no previous research has investigated the ERN and the Pe using temporal ordinal comparison tasks (e.g., Coull et al., 2004; Harrington et al., 2010; Rao et al., 2001; Van Rijn et al., 2011 - see also Larson and Clayson, 2011).

\section{$7 \quad$ Effects of Dopamine-related Error Processing on Interval Timing}

Like all errors, errors associated with interval timing should be followed by an error-correction signal from the DA system. This error correction signal 
represents the DA system's response to negative feedback. Even in the case where no feedback follows correct or incorrect responses, the ERN may still occur in situations where the experimental design allows for storage of the relevant stimuli in reference memory (see Cicchini et al., 2012). Consequently, when participants are trained to compare two sequentially presented signal durations in order to determine which signal is longer (Coull et al., 2004, 2012, 2013) the base durations are likely encoded during the early training trials and stored in the participant's reference memory for the rest of the session (Lustig and Meck, 2005; Matell and Meck, 2004). Thus, the cortico-striatal circuits may provide a form of "endogenous" feedback by comparing the current clock reading with previous durations stored in temporal memory and subsequently generate an ERN when judgments/responses do not match with those values previously stored in memory - this despite the fact that the judgment could have been made based upon the information presented within the current trial (Galea et al., 2012; Gu and Meck, 2011). The use of this type of statistical analysis of previous trials in the control of timing behavior on the current trial provides strong support for models based Bayesian decision theory (Körding and Wolpert, 2006).

In an unpublished study, we examined the effects of PD and dopaminergic medication on interval timing and error processing using an ordinal comparison procedure with hue and duration stimulus dimensions (Chen, 2007; see also Coull et al., 2004, 2012, 2013; Gu and Meck, 2011; Harrington et al., 2010). Psychophysical and EEG/ERP data were collected in PD patients ON and OFF dopaminergic medication. Based on previous studies of the ERN, the ERN was hypothesized to be present following both correct and incorrect responses, with a less negative ERN amplitude predicted for correct responses compared to incorrect responses (Beste et al., 2006, 2007; Falkenstein et al., 2005). Based on the refined mismatch theory, participants with increased DA levels were hypothesized to show higher ERN amplitudes. Thus, PD patients tested ON medication were hypothesized to show more negative ERN amplitudes compared to PD patients tested OFF medication. As predicted, we observed a reliable diminution in the ERN for PD patients tested OFF their dopaminergic medication compared to when these same patients were tested ON medication. In contrast, there were no consistent changes in the Pe as a function of dopaminergic medication in PD patients as illustrated in Figure 11.6. These results confirm the previous findings of Falkenstein et al. $(2001,2005)$ and support the view that deficient error processing may underlie the distortions in temporal memory observed in the PD-migration effects reviewed above. 


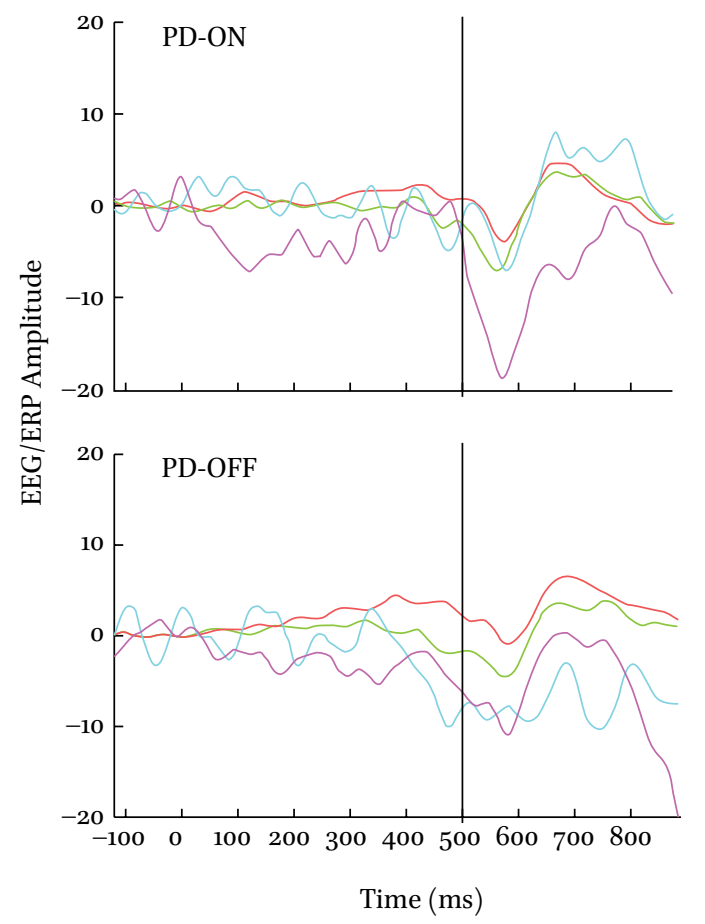

FIGURE 11.6 Ordinal comparison task using hue and duration stimulus combinations in the manner of Coull et al. (2004). The duration data presented here represent the grand average response-locked event-related potentials (ERPS) at electrode Fz after correct left-handed (green) and right-handed (red) responses and incorrect left-handed (magenta) and right-handed (cyan) responses in patients with Parkinson's disease (PD) in the ON and OFF medication states. The o-ms time point represents the time of the "shorter" or "longer" classification (key press) determined by a left-handed or right-handed response. The vertical line represents the peak latency of the error related negativity $(E R N)$ in the PD patients. The ERN is smaller in the OFF medication state than in the ON medication state for the left-handed (magenta) incorrect response with no difference in the Pe between conditions (see Falkenstein et al., 2001, 2005). Interestingly, no handedness/lateralization effects were observed for the hue condition in the PD patients as a function of the ON/OFF states.

\section{Vierordt's Law and the Migration of Temporal Memories}

Subjective time can be affected by various factors, including memory load and temporal context (Buhusi and Meck, 2005, 2009a,b; Buhusi et al., 2002; Grondin, 2005; Jazayeri and Shadlen, 2010; Merchant et al., 2008b). For example, when participants are presented with a range of signal durations and are then asked 
to reproduce those durations they tend to bias their reproductions towards the mean of the distribution of signal durations by overestimating "short" durations and underestimating "long" durations - a relationship known as Vierordt's law (Gu and Meck, 2011; Lejeune and Wearden, 2009; Mamassian and Landy, 2010). Furthermore, when auditory and visual signals are intermixed within a session, participants frequently overestimate auditory signals and underestimate visual signals of equivalent duration (Cheng et al., 2008, 2011; Penney et al., 2000, 2005). The range and modality of experienced signal durations are known to contribute to distortions in time estimation. Moreover, both of these phenomena have been hypothesized to be related to "memory-mixing" whereby similar clock readings are categorized into a limited number of memory distributions with the decision process involving a comparison of the current clock reading with a sample taken from each of these distributions (Gamache and Grondin, 2010; Gu and Kukreja, 2011; Gu and Meck, 2011; Klapproth, 2009; Lustig and Meck, 2011; Penney et al., 1998, 2008). For example, if auditory signals drive the internal clock faster (on average) than visual signals and memory distributions for a particular target duration are a mixture of these "shorter" visual and "longer" auditory clock readings, then auditory signals will have an increased probability of being judged "long" relative to visual signals of an equivalent physical duration. Alternatively, "memory-mixing" can occur when different standard durations used within the same temporal context (e.g., test session) are combined into a single memory distribution, rather than separate memory distributions being maintained for each standard. As a consequence of the temporal context, "memory-mixing" can produce distortions in scaling of duration that are consistent with Vierordt's law and may be modifiable by training conditions (e.g., blocking of different standards or signal modalities and/or by providing feedback in order to encourage the formation of separate rather than mixed-memory distributions). This sort of "memorymixing" is distinct from the time-order error, which refers to the influence of the order of presentation on the comparison of successively presented stimuli (see Allan, 1979; Brown et al., 2005; Eisler et al., 2008; Grondin, 2010). As described above, the mixing of unimodal stimuli in memory as a function of temporal context is consistent with Vierordt's law (Gu and Meck, 2011; Hairston and Nagarajan, 2007; Lejeune and Wearden, 2009; Mamassian and Landy, 2010), and the tendency for overestimating shorter durations and underestimating longer durations is one of the most robust temporal phenomena exhibited under a variety of conditions ( $\mathrm{Gu}$ and Meck, 2011; Jazayeri and Shadlen, 2010; Lejeune and Wearden, 2009). However, the exact mechanism underlying this "memory-mixing" effect and whether it can account for all of the PD-migration effects observed in the "encode/decode" paradigm is currently unknown. 
A recent series of investigations of temporal context has shown that a Bayesian model can explain the biased performance of participants when they are asked to reproduce or compare a recently presented signal duration (Cicchini et al., 2012; Gu and Meck, 2011;Jazayeri and Shadlen, 2010). This model incorporates the knowledge of the distribution of previous signal durations into the perception of the current signal duration, thus biasing the reproduction of the current interval towards the mean of the distribution. In this model, it is hypothesized that a tradeoff exists between accuracy and precision such that the distribution of signal durations (i.e., temporal context) is used to optimize performance by reducing temporal uncertainty at the cost of temporal accuracy. In this case, the implicit knowledge of the underlying distribution from which a sample is drawn would be useful when the current clock reading is uncertain due to the effects of noise and/or inattention, such as that which may result from dopaminergic drug administration (e.g., Lake and Meck, 2013) and/or neurological conditions that may affect dopaminergic function such as attention deficit hyperactivity disorder, Huntington's disease, PD, or obsessive compulsive disorder (e.g., Allman and Meck, 2012; Beste et al., 2007; Conners et al., 1996; Gu and Kukreja, 2011; Gu et al., 2008, 2011; Levin et al., 1996, 1998; Meck, 2005). This explains how the intermixing of a previous trial's signal durations with the perception of the current trial's signal duration could bias performance. Under certain conditions, however, this statistical analysis can provide an efficient strategy for reducing variability in the presence of high levels of uncertainty or noise (Cain et al., 2012; De Ridder et al., 2012). At present, this hypothesis is mainly supported by computer simulation rather than experimental results from behavioral or neurobiological studies.

Although the "memory-mixing" effect that we have described is highly reliable, large individual differences have been reported (Gu and Meck, 2011). Interestingly, healthy adult participants with a relatively high degree of "memory-mixing" display relatively low variation in the reaction times for their response classifications, whereas the opposite is true for participants with a low degree of "memory-mixing." Indeed, the "memory-mixing" index was negatively correlated with the observed variability in reaction time across participants. Although it is uncertain why variability in reaction time should be related to the level of "memory-mixing," one possibility is that participants who pay less attention to feedback utilize a more automatic and less variable process of sampling from a "mixed memory" distribution rather than engaging the more variable process of maintaining separate distributions under conditions of high temporal uncertainty (Gu and Meck, 2011). The observation of reliable individual differences in "memory-mixing" begs the question as to whether it is possible to increase or decrease the degree of "memory-mixing" 
by experimental manipulation. One can assume that if the degree of attention given to the encoding of the standard duration contributes to variation in the degree of "memory-mixing," then it would be possible to systematically alter the degree of "memory-mixing." Reward cues have been shown to increase the encoding of information through the activation of mesolimbic DA systems (Adcock et al., 2006; Koepp et al., 1998) and feedback tied to this dopaminergic system has been shown to improve the accuracy and precision of timing functions (Coull et al., 2011, 2012, 2013; Lustig and Meck, 2005; Malapani et al., 1998b; Meck, 2005). The assumption is that this feedback aids in the formation of modality-specific or standard-duration specific memory representations within the context of the psychophysical procedures described above. In order to determine whether feedback can affect the degree of "memory-mixing," participants were presented the word "WRONG" on the computer screen along with an aversive buzzer sound when they made an incorrect response and presented the word "CORRECT" along with a pleasant bell sound when they made a correct response. Following the implementation of this feedback procedure, some participants showed a dramatically reduced "memory-mixing" effect in ordinal comparison procedures ( $\mathrm{Gu}$ and Meck, 2011). It will be necessary to conduct additional studies in order to determine the most reliable and effective form of feedback (e.g., valence and intensity) for the modulation of "memory-mixing" as a function of development (Allman et al., 2012; DroitVolet and Meck, 2007) and different patient populations (Allman and Meck, 2012; Gu et al., 2008, 2011).

Previous work has shown that participants readily adjust to the underlying statistics involving the distributions of event durations in order to optimize the accuracy of their timed responses. This behavior is consistent with the development of a representation of the experimentally presented distribution of signal durations (the prior) and the calculation of the error (loss function) as predicted by Bayesian integration (Acerbi et al., 2012; Miyazaki et al., 2005). Within the Bayesian framework, the responses of an ideal observer depend on the manner in which these internal representations encode the complexity of the underlying distributions of signal durations (e.g., uniform to highly skewed or bimodal distributions). It has recently been shown that duration reproduction times are affected by both the underlying distribution of signal durations as well as the type and probability of corrective feedback (Acerbi et al., 2012; Sohn and Lee, 2013) - although the tracking of the distribution becomes more 
difficult with higher-order statistical features (e.g., kurtosis and multimodality). Overall, these findings are in good agreement with a performanceoptimizing Bayesian observer and actor model of decision-making suggesting that humans and other animals are sensitive to the temporal statistics of virtually all of the information presented within a specific temporal context. This statistical information influences the determination of temporal judgments even in cases where the current trial may simply require the reproduction of a signal duration that was presented to the participant just moments before the "go" signal to respond on the current trial (Acerbi et al., 2012; Cicchini et al., 2012; Jazayeri and Shadlen, 2010). Moreover, a Bayesian observer model, in which the subjective duration of an event is determined jointly by a prior and a likelihood function for timing, captures the temporal dynamics of accuracy and precision in a manner suggesting that the width of the prior, not the likelihoods, gradually decreases over training sessions, again indicating the relevance of prior knowledge for interval timing (Sohn and Lee, 2013).

Based on these prior arguments, a Bayesian-inspired model was used to simulate duration reproductions in the PI procedure using $7^{-}$and 14-s target durations (Lustig and Meck, 2005) as well as 8- and 21-s target durations (Malapani et al., 1998b) as illustrated in Figure 11.7 and Table 11.2. The model is designed to reduce reproduction errors under conditions where the time base is noisy as a result of a slowing in the mean clock speed and increased variability in its regulation - possibly as a result of DA deficiency and/or reduction in feedback. This is done by incorporating an adaptable central tendency prior as a probability density function centered at the mean of the distribution of target durations presented within all session blocks using the mean and standard deviation in the manner of Cicchini et al. (2012). The model did not associate different target durations with different discriminative stimuli because this feature was not present in the experimental procedures used for any of the PI data sets under study (e.g., Lustig and Meck, 2005; Malapani et al., 1998b, 2002; Rakitin et al., 1998, 2005). In this sense, the current model is a simplified version of the Bayesian least squares model used by Jazayeri and Shadlen (2010) and doesn't assume that all information is stored from previous trials and used to determine the prior even though this level of detail has been used in previous descriptions of interval timing (see Gibbon and Church, 1984, 1990, 1992).

Following Cicchini et al. (2012), we simulated the Bayesian model of estimated durations by incorporating information of previous target durations used within the same temporal context and a measurement of current trial's target duration. In this Bayesian model, the estimation of the current duration corresponds with the posterior distribution and this was calculated by combining the likelihood and prior functions, which represent the sensory 

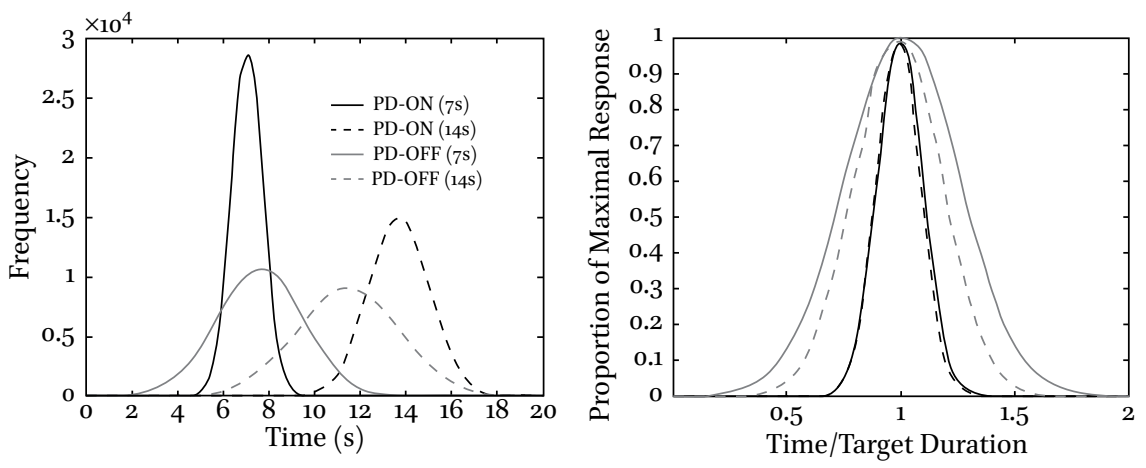

FIGURE 11.7 Bayesian simulations of 7-and 14-s target durations in the peak-interval procedure for Parkinson's disease (PD) patients tested ON and OFF dopaminergic medication. Weber fractions for sensory measurement $(W m)$ were set as 0.1 in $P D$ ON medication state and 0.3 in PD-OFF medication state. Simulation of 200,000 trials demonstates veridical timing (i.e., response functions centered on the target durations) in the PD-ON medication state and a high degree of migration (i.e., overestimation of the shorter duration and underestimation of the longer duration-Vierordt's law) for the 7-and 14-s target durations in the PD-OFF medication state (left panel). Scaled distribution functions, i.e., proportion of maximal responding plotted on a relative time scale (right panel), show that the estimations of 7-and 14-s target durations superimpose on top of each other in the PD-ON medication state. In contrast, the response function for the 14-s target duration shows a narrower dispersion compared to the response function for the 7-s target duration in the PD-OFF medication state, indicating a violation of scalar property of interval timing (see Hinton and Rao, 2004; Malapani et al., 1998; Rakitin et al., 1998).

measurement of the current target duration and the memory distribution of the previous target durations, respectively. The likelihood function was modeled as a Gaussian distribution with mean Ts (current target duration) and standard deviation (s.d.) WmTs - the measurement Weber fraction (Wm) multiplied by the target duration (Ts). According to these assumptions, increased variation will be correlated with the target duration(s), which corresponds to the scalar property of interval timing (Gibbon et al., 1984; Jazayeri and Shadlen, 2010). In addition, the $\mathrm{Wm}$ values for the $\mathrm{PD}-\mathrm{ON}$ and $\mathrm{PD}$-OFF medication states were set 0.1 and 0.3 , respectively, in order to represent the increased noise observed in sensory measurement and/or uncertainty as a function of DA depletion.

The prior function was defined as a Gaussian distribution centered at the mean of the previous target duration(s), and the s.d. (width) for each target duration was calculated to optimize performance considering precision and accuracy. In other words, the optimal s.d. of the prior was set to minimize the 
TABLE 11.2 Bayesian model predictions for peak-interval measurements.

\begin{tabular}{|c|c|c|c|c|c|c|}
\hline \multirow[b]{2}{*}{7 and $14 \mathrm{~s}$} & \multicolumn{2}{|c|}{$\begin{array}{l}\text { Measurement } \\
\left(\mathrm{Wm} \_O N=0.1 ;\right. \\
\text { Wm_OFF = o.3 })\end{array}$} & \multicolumn{2}{|c|}{$\begin{array}{l}\text { Reproduction } \\
(\text { Fixed Wp = 0.1) }\end{array}$} & \multicolumn{2}{|c|}{$\begin{array}{l}\text { Reproduction } \\
(\text { Wp_ON = o.1; } \\
\text { Wp_OFF = o.3) }\end{array}$} \\
\hline & Peak & $\mathrm{CV}$ & Peak & $\mathrm{CV}$ & Peak & $\mathrm{CV}$ \\
\hline $\mathrm{ON}(7 \mathrm{~s})$ & 7.07 & 0.10 & 7.07 & 0.14 & 7.07 & 0.14 \\
\hline $\mathrm{ON}(14 \mathrm{~s})$ & 13.89 & 0.10 & $13 \cdot 38$ & 0.14 & 13.38 & 0.14 \\
\hline $\mathrm{OFF}(7 \mathrm{~s})$ & $7 \cdot 5^{8}$ & 0.25 & $7 \cdot 5^{8}$ & 0.27 & 6.57 & 0.40 \\
\hline OFF (14s) & 11.62 & 0.19 & 11.36 & 0.22 & 10.86 & 0.36 \\
\hline 8 and $21 \mathrm{~s}$ & Peak & $\mathrm{CV}$ & Peak & $\mathrm{CV}$ & Peak & $\mathrm{CV}$ \\
\hline $\mathrm{ON}(8 \mathrm{~s})$ & 8.08 & 0.10 & 8.08 & 0.14 & 8.08 & 0.14 \\
\hline $\mathrm{ON}(21 \mathrm{~s})$ & 20.61 & 0.10 & 20.61 & 0.14 & 20.61 & 0.14 \\
\hline $\mathrm{OFF}(8 \mathrm{~s})$ & 8.48 & 0.27 & 8.08 & 0.29 & 6.87 & 0.41 \\
\hline OFF (21s) & 17.78 & 0.26 & 17.78 & 0.28 & 16.57 & 0.40 \\
\hline
\end{tabular}

Peak = peak time - mode of timing functions obtained from the peak-interval procedure with 7- and 14-s target durations or 8- and 21-s target durations for Parkinson's disease patients tested $\mathrm{ON}$ and $\mathrm{OFF}$ dopaminergic medication. $\mathrm{CV}=$ coefficient of variation - defined as the ratio of the standard deviation of the peak functions to the mean of the function as represented by the obtained peak time. $\mathrm{Wm}=$ Weber fraction for the measurement phase of timing. $\mathrm{Wp}=$ Weber fraction for the production phase of timing.

mean squared error (MSE), which is equal to the sum of the variance and the squared bias of the estimated target duration. In terms of these calculations, the width of the prior gets narrower as the value of WmTs get larger. For example, the 14 -s target duration in the PD-OFF medication state $(\mathrm{TsWm}=14 * 0.3=4.2$; prior width $=2.62$ ) has a much narrower prior width compared to the 7 -s target duration in PD-ON medication state $\left(\mathrm{TsWm}=7^{*} 0.1=0.7\right.$; prior width $\left.=4.9\right)$. In addition, if the temporal difference between the target durations gets smaller, the prior width also gets narrower. Therefore, the function for 7 - and 14-s target durations will be more affected by their prior compared to that of 8- and 21-s target durations. The narrower width (s.d.) of the prior in those cases may indicate that the duration estimation relies more on the memory distribution of previous target durations compared to the sensory measurement of the current target duration. By relying more on the memory of previous target durations, performance will result in lower accuracy (biased toward the mean), but higher precision in the reproduced duration than would otherwise be possible given the level of uncertainly in the DA-depleted, PD-OFF medication state. 
These estimated likelihood and prior functions were combined to determine the posterior function which corresponds to the distribution of estimated durations according to the Bayes' rule (Cicchini et al., 2012; Sohn and Lee, 2013). Simulation of 200,000 trials using 7 - and 14-s target durations from the calculated posterior in PD-ON/OFF medication states according to the methods described by $\mathrm{Gu}$ et al. (2015) shows that the degree of migration toward the mean was increased in the PD-OFF medication state as illustrated in Figure 11.7. The estimated peak times for the two target durations were 7.07 and $13.89 \mathrm{~s}$ in the PD-ON medication state, and 7.58 and $11.62 \mathrm{~s}$ in the PD-OFF medication state - showing nearly veridical timing in the $\mathrm{ON}$ state and the "PD migration" effect in the OFF medication state. In addition to the migration of peak times, the CVs for the two target durations were reliably different from each other in PD-OFF medication state ( 0.25 for $7 \mathrm{~s}$ and 0.19 for $14 \mathrm{~s}$ ) - more similar to counting than timing processes and a clear violation of the scalar property of interval timing (Hinton and Rao, 2004; Malapani et al., 1998b; Rakitin et al., 1998). In contrast, the Cv's were observed to be constant around 0.1 for both the 7-and 14-s target durations in the in PD-ON medication state. Given the high levels of uncertainty in sensory measurement due, in part, to a slowed clock in the PD-OFF medication state, participants rely more on the previous target durations when making temporal judgments. By doing so, the variation in duration estimation is reduced at the cost of estimation biases especially at longer target durations. Consequently, the violation of the scalar property of interval timing shown in the current Bayesian-model simulations is best understood as the by-product of incorporating prior information into the sensory measurement (Acerbi et al., 2012; Cicchini et al., 2012; Jazayeri and Shadlen, 2010; Shi et al., 2013).

The results of our simulations indicate a pattern of responding compatible with the observed PD-migration effects (e.g., Malapani et al., 1998b, 2002; Rakitin et al., 2005, 2008) as well as Vierordt's law (Gu and Meck, 2011; Lejeune and Wearden, 2009). The extent of the observed migration effect varied as a function of a parameter estimate of temporal precision, which can be related to the severity of DA depletion in normal aging and PD-ON/OFF medication states and/or the amount and type of feedback provided for responding (Frank, 2005; Lustig and Meck, 2005; Pessiglione et al., 2006; Volkow et al., 1998). As a consequence, this estimate of temporal precision can be viewed as similar to the differences observed for modality (auditory vs. visual) and musical training (controls vs. percussionists vs. strings) in the Bayesian-model simulations reported by Cicchini et al. (2012). These results suggest that drummers may be particularly sensitive to distortions in timing and time perception associated with PD and other types of basal ganglia dysfunction (Bilger, 2011). This Bayesian account is consistent with 
recent proposals suggesting that DA levels modulate the reliability and precision of sensory information. As a consequence, PD patients trained or tested in the OFF medication state have low DA levels and are therefore less confident about incoming sensory information and more reliant on top-down predictions (Galea et al., 2012). The major conclusion is that the underlying Bayesian likelihood function combined with a prior representing the distribution of target durations can be used to account for the migration effects observed as a function of normal aging and PD. Moreover, assuming that variability in measurement and production (e.g., clock and motor variability) increases in the PD-OFF medication condition, produces not only migration, but also a violation of the scalar property consistent with the observations from PD patients tested ON and OFF their DA replacement therapy (Malapani et al., 1998b, 2002).

Since the PD-migration effect was first identified by Malapani and colleagues (Malapani et al., 1998b), we now know that this phenomenon is the result of a DA-dependent dysfunction of retrieving temporal memories as opposed to a problem with encoding (Malapani et al., 2002). Moreover, although migration is observed in healthy older adults, it is of a smaller magnitude and shows a different pattern of variability in that the scalar property of interval timing is not violated as it is in PD patients taken off of their DA replacement therapy (Rakitin et al., 2005; Rakitin and Malapani, 2008). We also know that the PDmigration effect may be lateralized to the right hemisphere (Koch et al., 2005) and that deep brain stimulation of the sub-thalamic nucleus can reduce or increase these effects depending upon the frequency (and perhaps area) of stimulation (Koch et al., 2004; Wojtecki et al., 2011). Moreover, the size of PDmigration effect is modulated both by feedback and exogenously administered drugs thought to increase the effective level of DA - suggesting a common mechanism for these effects in terms of error processing and temporal precision ( $\mathrm{Gu}$ et al., 2015). Taken together, these findings are accounted for using a Bayesian model that assumes that increases in temporal uncertainty associated with PD are compensated for by combining the memories from multiple durations in order to be more accurate in the mean of the interval(s) being timed rather than trying to identify the specific target durations that form the distribution (Gu and Meck, 2011, 2013; Shi et al., 2013; also see Cicchini et al., 2012; Griffiths et al., 2008; Knill and Pouget, 2004). In this manner DA-deficient individuals are able to optimize performance by reducing temporal uncertainty at the cost of temporal accuracy - thus, 
demonstrating the types of temporal distortions presented in clinical situations involving the psychopathological mind (e.g., Allman and Meck, 2012; Allman et al., 2012, 2014a,b).

\section{References}

Acerbi,Luigi,Daniel M.Wolpert, and Sethu Vijayakumar. 2012.“Internal Representations of Temporal Statistics and Feedback Calibrate Motor-Sensory Interval Timing" PLoS Computational Biology 8(11): e1002771.

Adcock, R. Alison et al. 2006. "Reward-Motivated Learning: Mesolimbic Activation Precedes Memory Formation” Neuron 50: 507-17.

Agostino, Patricia V., Diego A. Golombek, and Warren H. Meck. 2011. "Unwinding the Molecular Basis of Interval and Circadian Timing" Frontiers in Integrative Neuroscience, 5: 64. Agostino, P.V. et al. 2013. "Acquisition of Response Thresholds for Timed Performance is Regulated by a Calcium-Responsive Transcription Factor, CaRF" Genes, Brain and Behavior 12: 633-44.

Allan, Lorraine G. 1979. "The Perception of Time" Perception and Psychophysics 26: 340-54. Allman, Melissa J., and Warren H. Meck. 2012. "Pathophysiological Distortions in Time Perception and Timed Performance" Brain 135: 656-77.

Allman, Melissa J., Kevin A. Pelphrey, and Warren H. Meck. 2012. "Developmental Neuroscience of Time and Number: Implications for Autism and Other Neurodevelopmental Disabilities" Frontiers in Integrative Neuroscience, 6: 7.

Allman, Melissa J. et al. 2014a. "Properties of the Internal Clock: First- and SecondOrder Principles of Subjective Time" Annual Review of Psychology 65: 743-71.

Allman, Melissa J., Bin Yin, and Warren H. Meck. 2014b. "Time in the Psychopathological Mind" In Subjective Time:The Philosophy, Psychology, and Neuroscience of Temporality, edited by Valtteri Arstila, and Dan Lloyd, 637-54. Cambridge, MA: MIT Press.

Artieda J. et al. 1992. "Temporal Discrimination is Abnormal in Parkinson's Disease" Brain 115: 199-210.

Balci, Fuat et al. 2009. "Timing Deficits in Aging and Neuropathology" In Animal Models of Human Cognitive Aging, edited by Jennifer L. Bizon, and Alisa Woods, 161-201. Totowa, NJ: Humana Press.

Benecke, R. et al. 1986. "Performance of Simultaneous Movements in Patients with Parkinson's Disease" Brain 109: 739-57.

Beste, Christian et al. 2006. "Error Processing in Huntington's Disease" PLoS ONE 1(1): e86.

Beste, Christian et al. 2007. “Time Processing in Huntington's Disease: A Group-Control Study" PLOS ONE 2(12): e1263.

Bilger, Burkhard. 2011. "The Possibilian:What a Brush with Death Taught David Eagleman About the Mysteries of Time and the Brain" The New Yorker 25 April, 54-6o, 62-65. 
Bloxham, C.A., D.J. Dick, and M. Moore. 1987. "Reaction Times and Attention in Parkinson's Disease" Journal of Neurology, Neurosurgery, and Psychiatry 50: 1178-83.

Brown, P. 2003. "Oscillatory Nature of Human Basal Ganglia Activity: Relationship to the Pathophysiology of Parkinson's Disease" Movement Disorders 18: 357-63.

Brown, R.G., and C.D. Marsden. 1988. "Internal Versus External Cues and the Control of Attention in Parkinson's Disease" Brain 111: 323-45.

Brown, Gordon D.A. et al. 2005. "Identification and Bisection of Temporal Durations and Tone Frequencies: Common Models for Temporal and Nontemporal Stimuli" Journal of Experimental Psychology: Human Perception and Performance 31: 919-38.

Brown, Peter et al. 2001. "Dopamine Dependency of Oscillations Between Subthalamic Nucleus and Pallidum in Parkinson's Disease" Journal of Neuroscience 21: 1033-8.

Bueti, Domenica, and Emiliano Macaluso. 2011. "Physiological Correlates of Subjective Time: Evidence for the Temporal Accumulator Hypothesis” NeuroImage 57: 1251-63.

Buhusi, Catalin V. 2003. "Dopaminergic Mechanisms of Interval Timing and Attention" In Functional and Neural Mechanisms of Interval Timing, edited by Warren H. Meck, 317-38. Boca Raton, FL: CRC Press.

Buhusi, Catalin V., and Warren H. Meck. 2002. "Differential Effects of Methamphetamine and Haloperidol on the Control of an Internal Clock" Behavioral Neuroscience 116: 291-7.

- 2005. "What Makes us Tick? Functional and Neural Mechanisms of Interval Timing" Nature Reviews Neuroscience 6: 755-65.

- 2009a. "Relative Time Sharing: New Findings and an Extension of the Resource Allocation Model of Temporal Processing” Philosophical Transactions of the Royal Society - London B 364: 1875-85.

- 20ogb. "Relativity Theory and Time Perception: Single or Multiple Clocks?" PLoS ONE 4(7): e6268.

Buhusi, Catalin V., and Sorien A. Oprisan. 2013. "Time-Scale Invariance as an Emergent Property in a Perceptron with Realistic, Noisy Neurons" Behavioural Processes 95: 60-70.

Buhusi, Catalin V., Aya Sasaki, and Warren H. Meck. 2002. "Temporal Integration as a Function of Signal/Gap Intensity in Rats (Rattus norvegicus) and Pigeons (Columba livia)" Journal of Comparative Psychology 116: 381-90.

Cain, Matthew S. et al. 2012. "A Bayesian Optimal Foraging Model of Human Visual Search" Psychological Science 23: 1047-54.

Castner, Stacy A., Graham V. Williams, and Patricia S. Goldman-Rakic. 20oo. "Reversal of Antipsychotic-Induced Working Memory Deficits by Short-Term Dopamine D1 Receptor Stimulation" Science 287: 2020-2.

Chen, Kelsey. 2007. "Electrophysiological Components of Error Processing in Age and Parkinson's Disease” Honors thesis submitted to Duke University, unpublished.

Cheng, Ruey-Kuang, and Warren H. Meck. 2007. "Prenatal Choline Supplementation Increases Sensitivity to Time by Reducing Non-Scalar Sources of Variance in Adult Temporal Processing" Brain Research 1186: 242-54. 
Cheng, Ruey-Kuang, Christopher J. MacDonald, and Warren H. Meck. 20o6. "Differential Effects of Cocaine and Ketamine on Time Estimation: Implications for Neurobiological Models of Interval Timing" Pharmacology Biochemistry and Behavior 85: 114-22.

Cheng, Ruey-Kuang et al. 2008. "Prenatal Choline Availability Differentially Modulates Timing of Auditory and Visual Stimuli in Aged Rats" Brain Research 1237: 167-75.

Cheng, Ruey-Kuang et al. 2011. "Categorical Scaling of Duration as a Function of Temporal Context in Aged Rats" Brain Research 1381: 175-86.

Church, Russell M., Warren H. Meck, and John Gibbon. 1994. "Application of Scalar Timing Theory to Individual Trials" Journal of Experimental Psychology: Animal Behavior Processes 20: 135-55.

Cicchini, Guido Marco et al. 2012. "Optimal Encoding of Interval Timing in Expert Percussionists" Journal of Neuroscience 32: 1056-6o.

Conners, C.K. et al. 1996. "Nicotine and Attention in Adult Attention Deficit Hyperactivity Disorder (AD HD)" Psychopharmacology Bulletin, 32: 67-73.

Cools, Roshan et al. 2002. "Dopaminergic Modulation of High-Level Cognition in Parkinson's Disease: The Role of the Prefrontal Cortex Revealed by PET" Brain 125: 584-94.

Coull, Jennifer T. et al. 2004. "Functional Anatomy of the Attentional Modulation of Time Estimation" Science 30: 1506-8.

Coull, Jennifer T., Ruey-Kuang Cheng, and Warren H. Meck. 2011. "Neuroanatomical and Neurochemical Substrates of Timing" Neuropsychopharmacology Reviews 36: $3^{-25}$.

Coull, Jennifer T. et al. 2012. "Dopamine Precursor Depletion Impairs Timing in Healthy Volunteers by Attenuating Activity in Putamen and Supplementary Motor Area" Journal of Neuroscience 32: 16704-15.

Coull, Jennifer T. et al. 2013. "Dopaminergic Modulation of Motor Timing in Healthy Volunteers Differs as a Function of Baseline DA Precursor Availability" Timing and Time Perception 1: 77-98.

Cui, Xu. 2011. "Hyperbolic Discounting Emerges From the Scalar Property of Interval Timing" Frontiers in Integrative Neuroscience 5: 24.

De Ridder, Dirk, Sven Vannests, and Walter Freeman. 2012. "The Bayesian Brain:" Phantom Percepts Resolve Sensory Uncertainty" Neuroscience and Biobehavioral Reviews 44:4-15., in press. <http://dx.doi.org/10.1016/j.neubiorev.2012.04.001>.

De Vries, Meinou H. et al. 2010. "Increasing Dopamine Levels in the Brain Improves Feedback-Based Procedural Learning in Healthy Participants: An ArtificialGrammar-Learning Experiment" Neuropsychologia 48: 3193-7.

Droit-Volet, Sylvie, and Warren H. Meck. 2007. "How Emotions Colour Our Perception of Time" Trends in Cognitive Sciences 11: 504-13.

Dušek, Petr et al. 2012. "Abnormal Activity in the Precuneus During Time Perception in Parkinson's Disease: An fMRI Study" PLoS ONE 7(1): e29635. 
Eagleman, David M. 2008. "Human Time Perception and Its Illusions" Current Opinion in Neurobiology 18: 131-136.

Ehlis, Ann-Christine et al. 2005. "Monitoring of Internal and External Error Signals" Journal of Psychophysiology 19: 263-9.

Eisler, Hannes, Anna D. Eisler, A., and Åke Hellström. 2008. "Psychophysical Issues in the Study of Time Perception" In Psychology of Time, edited by Simon Grondin, 75109. Bingley: Emerald Group Publishing.

Eusebio, Alexandre, Hayriye Cagnan, and Peter Brown. 2012. "Does Suppression of Oscillatory Synchronisation Mediate Some of the Therapeutic Effects of DBS in Patients with Parkinson's Disease?" Frontiers in Integrative Neuroscience 6: 47.

Evarts, E.V., H. Teravainen, and D.B. Calne. 1981. "Reaction Time in Parkinson's Disease" Brain 104: 167-86.

Falkenstein, Michael et al. 2000. "ERP Components on Reaction Errors and Their Functional Significance: A Tutorial” Biological Psychology 51: 87-107.

Falkenstein, Michael et al. 2001. "Action Monitoring, Error Detection, and the Basal Ganglia: An ERP Study" NeuroReport 12: 157-61.

Falkenstein, Michael et al. 2005. "Error Processing in Parkinson's Disease: The Error Positivity (Pe)" Journal of Psychophysiology 19: 305-10.

Fearnley, Julian M., and Andrew J. Lees. 1991. “Ageing and Parkinson's Disease: Substantia Nigra Regional Selectivity" Brain 114: 2283-301.

Ferrandez, A.M. et al. 2003. "Basal Ganglia and Supplementary Motor Area Subtend Duration Perception: An fMRI Study" NeuroImage 19: 1532-44.

Fournet, Nathalie et al. 2000. "Working Memory Functioning in Medicated Parkinson's Disease Patients and the Effect of Withdrawal of Dopaminergic Medication" Neuropsychology 14: 247-53.

Frank, Michael J. 2005. "Dynamic Dopamine Modulation in the Basal Ganglia: A Neurocomputational Account of Cognitive Deficits in Medicated and Nonmedicated Parkinsonism" Journal of Cognitive Neuroscience 17: 51-72.

Galea, Joseph M. et al. 2012. “Action Reprogramming in Parkinson's Disease: Response to Prediction Error is Modulated by Levels of Dopamine" Journal of Neuroscience 32: $542-50$.

Gamache, Pierre-Luc, and Simon Grondin. 2010. "The Lifespan of Time Intervals in Reference Memory" Perception 39:1431-51.

Gibbon, John. 1992. "Ubiquity of Scalar Timing with a Poisson Clock" Journal of Mathematical Psychology 35: 283-93.

Gibbon, John, and Russell M. Church. 1984. "Sources of Variance in an Information Processing Theory of Timing" In Animal Cognition, edited by Herbert L. Roitblat, Thomas G. Bever, and Herbert S. Terrace, 465-88. Hillsdale, NJ: Erlbaum.

- 1990. "Representation of Time" Cognition 37: 23-54. 1992. "Comparison of Variance and Covariance Patterns in Parallel and Serial Theories of Timing” Journal of The Experimental Analysis of Behavior 57: 393-406. 
Gibbon, John, and Chara Malapani. 2001. "Neural Basis of Timing and Time Perception" Encyclopedia of Cognitive Science, 401-406.

Gibbon, John, Russell M. Church, and Warren H. Meck. 1984. "Scalar Timing in Memory" Annals of The New York Academy of Sciences 423: 52-77.

Gibbon, John et al. 1997. "Toward a Neurobiology of Temporal Cognition: Advances and Challenges" Current Opinion in Neurobiology 7: 170-84.

Griffiths, Thomas L., Charles Kemp, and Joshua B. Tenebaum. 2008. "Bayesian Models of Cognition" In CsmbridgeThe Handbook of Computational Psychology, edited by Ron Sun, 1-49. Cambridge, U K: Cambridge University Press.

Grondin, Simon. 2005. "Overloading Temporal Memory" Journal of Experimental Psychology: Human Perception and Performance 31: 869-79.

Grondin, Simon. 2010. "Timing and Time Perception: A Review of Recent Behavioral and Neuroscience Findings and Theoretical Directions" Attention Perception and Psychophysics 72: 561-82.

$\mathrm{Gu}$, Bon-Mi and Keshav Kukreja. 2011. "Obsessive-Compulsive Disorder and MemoryMixing in Temporal Comparison: Is Implicit Learning the Missing Link?” Frontiers in Integrative Neuroscience 5: 38 .

$\mathrm{Gu}$, Bon-Mi, and Warren H. Meck. 2011. "New Perspectives on Vierordt's Law: MemoryMixing in Ordinal Temporal Comparison Tasks" Lecture Notes in Computer Science 6789 LNAI: $67-78$.

Gu, Bon-Mi, Hedderik van Rijn, and Warren H. Meck. 2015. "Oscillatory Multiplexing of Neural Population Codes for Interval Timing and Working Memory" Neuroscience \& Biobehavioral Reviews 48: 160-85.

$\mathrm{Gu}$, Bon-Mi et al 2008. "Neural Correlates of Cognitive Inflexibility During TaskSwitching in Obsessive-Compulsive Disorder" Brain 131: 155-64.

$\mathrm{Gu}$, Bon-Mi et al. 2011. "Quinpirole-Induced Sensitization to Noisy/Sparse Periodic Input: Temporal Synchronization as a Component of Obsessive-Compulsive Disorder" Neuroscience 179: 143-50.

_. 2015. "Bayesian Models of Interval Timing and Distortions in Temporal Memory as a Function of Dopamine Levels in Young, Aged, and Parkinson's Disease Patients" Submitted.

Hallett, Mark, and Shahram Khoshbin. 1980. "A Physiological Mechanism of Bradykinesia" Brain 103: 301-14.

Hairston, Ilana S., and Srikantan S. Nagarajan. 2007. "Neural Mechanisms of the TimeOrder Error: An MEG study" Journal of Cognitive Neuroscience 19: 1163-74.

Harrington, Deborah L., Kathleen Y. Haaland, and Neal Hermanowicz.1998a. "Temporal Processing in the Basal Ganglia" Neuropsychology 12: 3-12.

Harrington, Deborah L., Kathleen Y. Haaland, and Robert T. Knight. 1998b. "Cortical Networks Underlying Mechanisms of Time Perception" Journal of Neuroscience 18: 1085-95. Harrington, Deborah L. et al. 2010. "Neural Modulation of Temporal Encoding, Maintenance, and Decision Processes" Cerebral Cortex 20: 1274-85. 
Harrington, Deborah L. et al. 2011a. "Neural Underpinnings of Distortions in the Experience of Time Across Senses" Frontiers in Integrative Neuroscience 5: 32.

Harrington, Deborah L. et al. 2011b. "Neurobehavioral Mechanisms of Temporal Processing Deficits in Parkinson's Disease” PLoS ONE 6(2): e17461.

Harrington, Deborah L. et al. 2014. "Dissociation of Neural Mechanisms for Intersensory Timing Deficits in Parkinson's Disease" Timing and Time Perception 2: 17-43.

Herrmann, Christoph S., MAtthias H.J. Munk, and Andreas K. Engel. 2004. "Cognitive Functions of Gamma-Band Activity: Memory Match and Utilization" Trends in Cognitive Sciences 8: 347-55.

Hinton, Sean C. 2003. "Neuroimaging Approaches to the Study of Interval Timing" In Functional and Neural Mechanisms of Interval Timing edited by Warren H. Meck, 419-38. Boca Raton, FL: CRC Press.

Hinton, Sean C., and Stephen M. Rao. 2004. "One-Thousand One...One-Thousand Two...': Chronometric Counting Violates the Scalar Property in Interval Timing” Psychonomic Bulletin and Review 11: 24-30.

Hinton, Sean C., and Warren H. Meck. 1997. "How Time Flies: Functional and Neural Mechanisms of Interval Timing" In Time and Behaviour: Psychological and Neurobiological Analyses. edited by Christopher M. Bradshaw and Elmer Szabadi, 409-57. New York: Elsevier.

Hinton, Sean C., and Warren H. Meck. 2004. "Frontal-Striatal Circuitry Activated by Human Peak-Interval Timing in the Supra-Seconds Range" Cognitive Brain Research, 21: $171-82$.

Höhn, Sophie et al. 2011. "Behavioral and In Vivo Electrophysiological Evidence for Presymptomatic Alteration of Prefronto-Striatal Processing in the Transgenic Rat Model for Huntington Disease" Journal of Neuroscience 31: 8986-97.

Holroyd, Clay B., and Michael G.H Coles 2002. "The Neural Basis of Human Error Processing: Reinforcement Learning, Dopamine, and the Error-Related Negativity" Psychological Review 109: 679-709.

Holroyd, Clay B. et al. 2002. "Spared Error-Related Potentials in Mild to Moderate Parkinson's Disease" Neuropsychologia 40: 2116-24.

Hutchison, William D. et al. 2004. "Neuronal Oscillations in the Basal Ganglia and Movement Disorders: Evidence From Whole Animal and Human Recordings" Journal of Neuroscience 24: 9240-3.

Ito, Junko, and Junko Kitagawa. 2006. "Performance Monitoring and Error Processing During a Lexical Decision Task in Patients with Parkinson's Disease” Journal of Geriatric Psychiatry and Neurology 19: 46-9.

Ivry, Richard B. 1996. "The Representation of Temporal Information in Perception and Motor Control" Current Opinion in Neurobiology 6: 851-7.

Ivry, Richard B., and R. Eliot Hazeltine. 1995. "Perception and Production of Temporal Intervals Across a Range of Durations: Evidence for a Common Timing Mechanism" Journal of Experimental Psychology: Human Perception and Performance 21: 3-18. 
Ivry, Richard B., and Steven W. Keele. 1989. "Timing Functions of the Cerebellum" Journal of Cognitive Neuroscience 1: $137-52$.

Ivry, R.B., S.W. Keele, and Diener, H.C. 1988. "Dissociation of the Lateral and Medial

Cerebellum in Movement Timing and Movement Execution" Experimental Brain Research 73: 167-80.

Jazayeri, Mehrdad, and Michael N. Shadlen. 2010. "Temporal Context Calibrates Interval Timing" Nature Neuroscience 13: 1020-6.

Jahanshahi, Marjan et al. 2006. "The Substantia Nigra Pars Compacta and Temporal Processing" Journal of Neuroscience, 26: 12266-73.

Jahanshahi, Marjan et al. 2010a. "Dopaminergic Modulation of Striato-Frontal

Connectivity During Motor Timing in Parkinson's Disease” Brain 133: 727-45.

Jahanshahi, Marjan et al. 2010b. "Medication Impairs Probabilistic Classification

Learning in Parkinson's Disease" Neuropsychologia 48: 1096-103.

Jones, Catherine R.G. et al. 2011. "Modeling Accuracy and Variability of Motor Timing in

Treated and Untreated Parkinson's Disease and Healthy Controls" Frontiers in Integrative Neuroscience, 5: 81.

Jones, Catherine R.G., and Marjan Jahanshahi. 2011. "Dopamine Modulates Striato-Frontal Functioning During Temporal Processing” Frontiers in Integrative Neuroscience, 5: 70.

Jones, Catherine R.G., and Marjan Jahanshahi. 2014. "Contributions of the Basal Ganglia

to Temporal Processing: Evidence from Parkinson's Disease" Timing and Time Perception, 2: 87-127.

Jones, Catherine R.G. et al. 2008. "Basal Ganglia, Dopamine and Temporal Processing: Performance on Three Timing Tasks On and Off Medication in Parkinson's Disease” Brain and Cognition 68: 30-41.

Jones, Catherine L. et al. 2011. “Under Pressure: Response Urgency Modulates Striatal and Insula Activity during Decision- Making under Risk” PLoS ONE 6(6): e2og42.

Jurkowski, Anita J., and Warren H. Meck. 2003. “Could Interval Timing Be Used as an Early Marker for PD?" Presentation given at the Udall Parkinson's Disease Research Center of Excellence Symposium, Duke University, Durham, NC, September 10-11, 2003.

Jurkowski, Anita J., and Mark Stacy. 2005. "Classification and Clinical Features of Movement Disorders" In Movement Disorders: Genetics and Models, edited by Mark S. LeDoux, 1-12. San Diego, CA: Elsevier Academic Press.

Jurkowski, Anita J., Elizabeth Stepp, and Steven A. Hackley. 2005a. "Variable Foreperiod Deficits in Parkinson's Disease: Dissociation Across Reflexive and Voluntary Behaviors" Brain and Cognition 58: 49-61.

Jurkowski, Anita J., Elizabeth Stepp, and Steven A. Hackley. 2002. "Dissociation in Reflexive and Voluntary Behaviors for Interval Timing in Aged and Parkinsonian Adults" Journal of Cognitive Neuroscience, S, 129-E37.

Jurkowski, Anita J. et al. 2005b. “Dopamine Levels in Young, Aged, and Parkinson's Adults Predict Behavioral and Electrophysiological Measures of Color and Time 
Perception" Program No. 329.47 Neuroscience 2005 Abstracts. Washington, D.C., Society for Neuroscience. Online.

Kawashima, Shoji et al. 2012. "Changes in Striatal Dopamine Release Associated with Human Motor-Skill Acquisition” PLoS ONE 7(2): e31728.

Keele, Steven et al. 1985. "Do Perception and Motor Production Share Common Timing Mechanisms? A Correlational Analysis” Acta Psychologica 6o: 173-91.

Kim, Namsoo et al. 2014. "Striatal Firing Rate Reflects Head Movement Velocity" European Journal of Neuroscience, 40: 3481-90.

Kim, Soyoun, and Daeyeol Lee. 2011. "Prefrontal Cortex and Impulsive Decision Making” Biological Psychiatry 69: 1140-6.

Klapproth, Florian. 2009. "Single-Modality Memory Mixing in Temporal Generalization: An Effect Due to Instructional Ambiguity" NeuroQuantology 7: 8-94.

Knill, David C., and Alexandre Pouget. 2004. "The Bayesian Brain: The Role of Uncertainty in Neural Coding and Computation for Perception and Action" Trends in Neuroscience 27: 712-9.

Koch, Giacomo et al. 2003. "Underestimation of Time Perception After Repetitive Transcranial Magnetic Stimulation" Neurology 60: 1844-6.

Koch, Giacomo et al. 2004. "Subthalamic Deep Brain Stimulation Improves Time Perception in Parkinson's Disease" NeuroReport, 15: 1071-3.

Koch, Giacomo et al. 2005. "Memory for Time Intervals is Impaired in Left HemiParkinson Patients" Neuropsychologia 43: 1163-7.

Koch, Giacomo et al. 2008. "Impaired Reproduction of Second But Not Millesecond Time Intervals in Parkinson's Disease" Neuropsychologia 46: 1305-13.

Koch, Giacomo Massimiliano Oliveri, and Carlo Caltagirone. 2009. "Neural Networks Engaged in Milliseconds and Seconds Time Processing: Evidence From Transcranial Magnetic Stimulation and Patients with Cortical or Subcortical Dysfunction" Philosophical Transactions of the Royal Society B 364: 1907-18.

Koepp, M.J. et al. 1998. "Evidence For Striatal Dopamine Release During a Video Game" Nature 393: 266-8.

Kolev, Vasil, Michael Falkenstein, and Juliana Yordanova. 2005. "Aging and Error Processing: Time-Frequency Analysis of Error-Related Potentials" Journal of Psychophysiology 19: 289-97.

Kotz, Sonia A., Michael Schwartze. 2011. "DIfferential Input of the Supplementary Motor Area to a Dedicated Temporal Processing Network: Functional and Clinical Implications" Frontiers in Integrated Neuroscience, 5, 86.

Körding, Konrad P., and Daniel M. Wolpert. 2006. "Bayesian Decision Theory in Sensorimotor Control" Trends in Cognitive Sciences 10: 319-26.

Lake, Jessica I., and Warren H. Meck. 2013. "Differential Effects of Amphetamine and Haloperidol on Temporal Reproduction: Dopaminergic Regulation of Attention and Clock Speed" Neuropsychologia 51: 284-92. 
Larson, Michael J., and Peter E. Clayson. 2011. "The Relationship between Cognitive Performance and Electrophysiological Indices of Performance Monitoring" Cognitive, Affective, \& Behavioral Neuroscience 11: 159-71.

Lee, Eun-Young et al. 2010. "Visual Working Memory Deficits in Patients With Parkinson's

Disease are Due to Both Reduced Storage Capacity and Impaired Ability to Filter Out Irrelevant Information" Brain 133: 2677-89.

Lejeune, Helga, and John H. Wearden. 2009. "Vierordt's The Experimental Study of the

Time Sense (1868) and its Legacy" European Journal of Cognitive Psychology 21: 941-6o.

Levin, E.D. et al. 1996. "Nicotine Effects on Adults with Attention-Deficit/Hyperactivity Disorder" Psychopharmacology 123: 55-63.

Levin, Edward D. et al. 1998. "Transdermal Nicotine Effects on Attention" Psychopharmacology 140: 135-41.

Lieberman, Philip et al. 1992. "Speech Production, Syntax Comprehension, and Cognitive Deficits in Parkinson's Disease" Brain and Language 43: 169-89.

Lustig, Cindy. 2003. "Grandfather's Clock: Attention and Interval Timing in Older Adults" In Functional and Neural Mechanisms of Interval Timing, edited by Warren H. Meck, 261-93. Boca Raton, FL: CRC Press.

Lustig, Cindy, and Warren H. Meck. 2001. "Paying Attention to Time as One Gets Older" Psychological Science 12: 478-84.

_. 2005. "Chronic Treatment with Haloperidol Induces Working Memory Deficits in Feedback Effects of Interval Timing” Brain and Cognition 58: 9-16.

. 2011. "Modality Differences in Timing and Temporal Memory Throughout the Lifespan" Brain and Cognition 77: 298-303.

Lustig, Cindy, Matthew S. Matell, and Warren H. Meck. 2005. "Not 'Just' a Coincidence: Frontal-Striatal Synchronization in Working Memory and Interval Timing" Memory 13: 441-8.

MacDonald, Christopher J., and Warren H. Meck. 2004. "Systems-Level Integration of Interval Timing and Reaction Time" Neuroscience and Biobehavioral Reviews 28: $747-69$.

-2005. "Differential Effects of Clozapine and Haloperidol on Interval Timing in the Supraseconds Range" Psychopharmacology 182: 232-44.

- 2006. "Interaction of Raclopride and Preparatory-Interval Effects on Simple Reaction-Time Performance" Behavioural Brain Research 175: 62-74.

MacDonald, Christopher J., Ruey-Kuang Cheng, and Warren H. Meck. 2012. "Acquisition of 'Start' and 'Stop' Response Thresholds in Peak-Interval Timing is Differentially Sensitive to Protein Synthesis Inhibition in the Dorsal and Ventral Striatum" Frontiers in Integrative Neuroscience 6: 10.

MacDonald, Christopher J. et al. 2014. "Retrospective and Prospective Views on the Role of the Hippocampus in Interval Timing and Memory for Elapsed Time" Timing and Time Perception 2: 51-61. 
Malapani, Chara, and Stephen Fairhurst. 2002. "Scalar Timing in Animals and Humans" Learning and Motivation 33: 156-76.

Malapani, Chara, and Brian C. Rakitin. (2003). "Interval Timing in the DopamineDepleted Basal Ganglia: From Empirical Data to Timing Theory" In Functional and Neural Mechanisms of Interval Timing, edited by Warren H. Meck, 485-514. Boca Raton, FL: CRC Press.

Malapani, Chara et al. 1994. "Impaired Simultaneous Cognitive Task Performance in Parkinson's Disease: A Dopamine Related Dysfunction” Neurology 44: 319-26.

Malapani, Chara et al. 1998a. "Cerebellar Dysfunction of Temporal Processing in the Seconds Range in Humans" Neuroreport, 9: 3907-12.

Malapani, Chara et al. 1998b. "Coupled Temporal Memories in Parkinson's Disease: A Dopamine-Related Dysfunction" Journal of Cognitive Neuroscience 10: 316-31.

Malapani, Chara, Bernard Deweer, and John Gibbon. 2002. "Separating Storage From Retrieval Dysfunction of Temporal Memory in Parkinson's Disease" Journal of Cognitive Neuroscience 13: 311-22.

Mamassian, Pascal, and Michael S. Landy. 2010. "It's That Time Again" Nature Neuroscience 13: 914-6.

Maricq, Andres V., and Russell M. Church. 1983. "The Differential Effects of Haloperidol and Methamphetamine on Time Estimation in the Rat" Psychopharmacology 79: 10-15.

Matell, Matthew S., and Warren H. Meck. 2000. "Neuropsychological Mechanisms of Interval Timing Behaviour" BioEssays 22: 94-103.

- 2004. "Cortico-Striatal Circuits and Interval Timing: Coincidence-Detection of Oscillatory Processes" Cognitive Brain Research 21: 139-70.

Matell, Matthew S., George R. King, and Warren H. Meck. 2004. “Differential Adjustment of Interval Timing by the Chronic Administation of Intermittent or Continuous Cocaine" Behavioral Neuroscience 118: 150-6.

Matell, Matthew S., Melissa Bateson, and Warren H. Meck. 2006. "Single-Trials Analyses Demonstrate That Increases in Clock Speed Contribute to the MethamphetamineInduced Horizontal Shifts in Peak-Interval Timing Functions" Psychopharmacology 188: 201-12.

Matell, Matthew S., Warren H. Meck and Miguel A.L. Nicolelis. 2003. "Interval Timing and the Encoding of Signal Duration by Ensembles of Cortical and Striatal Neurons" Behavioral Neuroscience 117: 760-73.

Matthews, William J., Warren H. Meck. 2014. "Time Perception: The Bad News and the Good" Wiley Interdisciplinary Reviews: Cognitive Science 5: 429-46.

Meck, Warren H. 1983. "Selective Adjustment of the Speed of Internal Clock and Memory Processes" Journal of Experimental Psychology: Animal Behavior Processes 9: 171-201.

1986. "Affinity for the Dopamine D2 Receptor Predicts Neuroleptic Potency in Decreasing the Speed of an Internal Clock" Pharmacology Biochemistry and Behavior 25: 1185-9. 
1988. "Hippocampal function is required for feedback control of an internal clock's criterion" Behavioral Neuroscience 102: 54-6o.

1996. "Neuropharmacology of Timing and Time Perception" Cognitive Brain Research 3: 227-42.

- 2002a. "Choline Uptake in the Frontal Cortex is Proportional to the Absolute Error of a Temporal Memory Translation Constant in Mature and Aged Rats" Learning and Motivation 33: 88-104.

- 2002b. "Distortions in the Content of Temporal Memory: Neurobiological Correlates" In Animal Cognition and Sequential Dehavior: Behavioral, Biological, and Computational Perspectives, edited by Stephen B. Fountain, Michael D. Bunsey, Joseph H. Danks, and Michael K. McBeath, 175-200. Boston, MA: Kluwer Academic Press.

2003. Functional and Neural Mechanisms of Interval Timing. Boca Raton, FL: CRC Press LLC.

-2005. "Neuropsychology of Timing and Time Perception" Brain and Cognition 58: $1-8$.

- 2006a. "Frontal Cortex Lesions Eliminate the Clock Speed Effect of Dopaminergic Drugs on Interval Timing” Brain Research 1108: 157-67.

- 2006b. "Neuroanatomical Localization of an Internal Clock: A Functional Link Between Mesolimbic, Nigrostriatal, and Mesocortical Dopaminergic Systems" Brain Research 1109: 93-107.

- 2006c. "Temporal Memory in Mature and Aged Rats is Sensitive to Choline Acetyltransferase Inhibition" Brain Research 1108: 168-75.

Meck, Warren H., and Aimee M. Benson. 2002. "Dissecting the Brain's Internal Clock: How Frontal-Striatal Circuitry Keeps Time and Shifts Attention" Brain and Cognition 48: $195^{-211 .}$

Meck, Warren H., and Chara Malapani. 2004. "Neuroimaging of Interval Timing" Cognitive Brain Research 21: 133-7.

Meck, Warren H., and Katherine E. Angell. 1992. "Repeated Administration of Pyrithiamine Leads to a Proportional Increase in the Remembered Durations of Events" Psychobiology 20: 39-46.

Meck, Warren H., and Russell M. Church. 1987. "Cholinergic Modulation of the Content of Temporal Memory" Behavioral Neuroscience 101: 457-64.

Meck, Warren H. et al. 2012a. "Gene-Dose Dependent Effects of Methamphetamine on Interval Timing in Dopamine-Transporter Knockout Mice” Neuropharmacology 62: 1221-9.

Meck, Warren H, Valérie Doyère, and Agnès Gruart. 2012b. "Interval Timing and TimeBased Decision Making" Frontiers in Integrative Neuroscience 6: 13.

Meck, Warren H., Russell M. Church, and Gary L. Wenk. 1986. "Arginine Vasopressin Inoculates Against Age-Related Increases in Sodium-Dependent High Affinity Choline Uptake and Discrepancies in the Content of Temporal Memory" European Journal of Pharmacology 130: 327-31. 
Meck, Warren H., Russell M. Church, and Matthew S. Matell. 2013. "Hippocampus, time, and memory - A Retrospective Analysis" Behavioral Neuroscience 127: 642-54.

Meck, Warren H., Fariba N. Komeily-Zadeh, and Russell M. Church. 1984. "Two-Step Acquisition: Modification of an Internal Clock's Criterion" Journal of Experimental Psychology: Animal Behavior Processes 10: 297-306.

Meck, Warren H., Trevor B. Penney, and Viviane Pouthas. 2008. "Cortico-Striatal Representation of Time in Animals and Humans" Current Opinion in Neurobiology 18: $145^{-52 .}$

Merchant, Hugo, Wilbert Zarco, and Luis Prado. 2007. "Do We Have a Common Mechanism for Measuring Time in the Hundreds of Millisecond Range? Evidence From Multiple-Interval Timing Tasks" Journal of Neurophysiology 99: 939-49.

Merchant, Hugo et al. 2008a. "Interval Timing and Parkinson's Disease: Heterogeneity in Temporal Performance" Experimental Brain Research 184: 233-48.

Merchant, Hugo et al. 2008b. "The Context of Temporal Processing Is Represented in the Multidimensional Relationships between Timing Tasks" PLoS ONE 3(9): e3169.

Merchant, Hugo, Deborah L. Harrington, and Warren H. Meck. 2013a. "Neural Basis of the Perception and Estimation of Time" Annual Review of Neuroscience 36 : $313-36$.

Merchant, Hugo et al. 2013b. "Interval Tuning in the Primate Medial Premotor Cortex as a General Timing Mechanism" Journal of Neuroscience 33: 9082-96.

Miyazaki, Makoto, Daichi Nozaki, and Yasoichi Nakajima. 2005. "Testing Bayesian Models of Human Coincidence Timing" Journal of Neurophysiology 94: 395-9.

Nagano-Saito Atsuko et al. 2008. "Dopamine Depletion Impairs Frontostrital Functional Connectivity During a Set-Shifting Task" Journal of Neuroscience 28: 3697-706.

Nakamura, Ryuichi, Hiroshi Nagasaki, and Hirotaro Narabayashi. 1978. "Disturbances of Rhythm Formation in Patients with Parkinson's Disease: Part 1. Characteristics of Tapping Response to the Period Signals" Perceptual and Motor Skills 46: 63-75.

Nichelli, Paolo, David Alway, and Jordan Grafman. 1996. "Perceptual Timing in Cerebellar Degeneration" Neuropsychologia 34: 863-72.

Nieuwenhuis, Sander et al. 2002. "A Computational Account of Altered Error Processing in Older Age: Dopamine and the Error-Related Negativity" Cognitive, Affective, and Behavioral Neuroscience 2: 19-36.

Nieuwenhuis, Sander et al. 2004. "Reinforcement-Related Brain Potentials from Medial Frontal Cortex: Origins and Functional Significance" Neuroscience and Biobehavioral Reviews 28: 441-8.

O'Boyle, Donald J., John S. Freeman, and Frederick W.J. Cody. 1996. “The Accuracy and Precision of Timing of Self-Paced, Repetition Movements in Subjects with Parkinson's Disease" Brain 119: 51-70.

Oprisan, Sorien A., and Catalin V. Buhusi. 2011. "Modeling Pharmacological Clock and Memory Patterns of Interval Timing in a Striatal Beat-Frequency Model with Realistic, Noisy Neurons" Frontiers in Integrative Neuroscience 5: 52. 
2013. "How Noise Contributes to Time-Scale Invariance of Interval Timing" Physical Review E 87: 052717.

Overbeek, Therese J.M., Sander Nieuwenhuis, and K. Richard Ridderinkhof. 2005. "Dissociable Components of Error Processing: On the Functional Significance of the Pe Vis-a-vis the ERN/Ne" Journal of Psychophysiology 19: 319-29.

Parker, K.L. et al. 2013. "Executive Dysfunction in Parkinson's Disease and Timing Deficits" Frontiers in Integrative Neuroscience 7: 75.

Pastor, M.A. et al. 1992. “Time Estimation and Reproduction is Abnormal in Parkinson's Disease" Brain 115: 211-25.

Penney, Trevor B. et al. 1998. "Memory Mixing in Duration Bisection" In Timing of Behavior: Neural, Psychological and Computational Perspectives, edited by David A. Rosenbaum, and Charles E. Collyer, 165-93. Cambridge, MA: MIT Press.

Penney, Trevor B., John Gibbon, and Warren H. Meck. 200o. "Differential Effects of Auditory and Visual Signals on Clock Speed and Temporal Memory" Journal of Experimental Psychology: Human Perception and Performance 26: 1770-87.

Penney, Trevor B. et al. 2005. "Interval-Timing Deficits in Individuals at High Risk for Schizophrenia" Brain and Cognition 58: 109-18.

Penney, Trevor B., John Gibbon, and Warren H. Meck. 2008. "Categorical Scaling of Duration Bisection in Pigeons (Columba livia), Mice (Mus musculus), and Humans (Homo sapiens)" Psychological Science 19: 1103-9.

Perbal, Séverine et al. 2005. "Effects of Internal Clock and Memory Disorders on Duration Productions and Reproductions in Patients with Parkinson's Disease" Brain and Cognition 58: 35-48.

Pessiglione, Mathias et al. 2006. "Dopamine-Dependent Prediction Errors Underpin Reward-Seeking Behavior in Humans" Nature 442: 1042-5.

Philiastides, Marios G. et al. 2011. "Causal Role of Dorsolateral Prefrontal Cortex in Human Perceptual Decision Making” Current Biology 21: 980-3.

Pouthas, Viviane, and Séverine Perbal. 2004. "Time Perception Depends on Accurate Clock Mechanisms as Well as Unimpaired Attention and Memory Processes" Acta Neurobiologiae Experimentalis 64: 367-85.

Rahnev, Dobromir, Hakwan Lau, and Floris P. de Lange. 2011. "Prior Expectation Modulates the Interaction Between Sensory and Prefrontal Regions in the Human Brain" Journal of Neuroscience 31: 10741-8.

Rakitin, Brian C., and Chara Malapani. 2008. "Effects of Feedback on Time Production Errors in Aging Participants" Brain Research Bulletin 75: 23-33.

Rakitin, Brian C., and Yaakov Stern. 2006. "Parkinson Disease" Encyclopedia of Cognitive Science, $45^{8-462 .}$

Rakitin, Brian C. et al. 1998. "Scalar Expectancy Theory and Peak-Interval Timing in Humans" Journal of Experimental Psychology: Animal Behavior Processes 24: 15-33. 
Rakitin, Brian C., Yaakov Stern, and Chara Malapani. 2005. "The Effects of Aging on Time Reproduction in Delayed Free-Recall" Brain and Cognition 58: 17-34.

Rakitin, Brian C. et al. 2006. "Single-Dose Levodopa Administration and Aging Independently Disrupt Time Production" Journal of Cognitive Neuroscience 18: 376-87.

Rao, Stephen M., Andrew R. Meyer, and Deborah L. Harrington. 2001. "The Evolution of Brain Activation During Temporal Processing" Nature Neuroscience 4: 317-23.

Ray, Debajyoti, and Peter Bossaerts. 2011. "Positive Temporal Dependence of the Biological Clock Implies Hyperbolic Discounting" Frontiers in Integrative Neuroscience 5: 2.

Roberts, Seth. 1981. "Isolation of an Internal Clock" Journal of Experimental Psychology: Animal Behavior Processes 7: 242-68.

—. 1993. "Use of Independent and Correlated Measures to Divide a TimeDiscrimination Mechanism Into Parts" Attention and performance, XIV, 589-610, MIT Press, Cambridge, MA.

Roy, Eric A. et al. 1993. "Movement Sequencing Disorders in Parkinson's Disease" International Journal of Neuroscience 73:183-94.

Rubin, David C. 1999. "Frontal-Striatal Circuits in Cognitive Aging: Evidence for Caudate Involvement" Aging, Neuropsychology, and Cognition 6: 241-59.

San Martín, Réne. 2012. "Event-Related Potential Studies of Outcome Processing and Feedback-Guided Learning" Frontiers in Human Neuroscience 6: 304.

Schirmer Annett. 2004. "Timing Speech: A Review of Lesion and Neuroimaging Findings" Cognitive Brain Research 21: 269-87.

Schultz, Wolfram. 1998. "Predictive Reward Signal of Dopamine Neurons" Journal of Neurophysiology 80: 1-27.

Schultz, Wolfram, and Anthony Dickinson. 2000. "Neuronal Coding of Prediction Errors" Annual Review of Neuroscience 23: 473-500.

Shea-Brown, Eric et al. 2006. "A Firing Rate Model of Parkinson's Deficits in Interval Timing" Brain Research 1070: 189-201.

Shi, Zhuanghua, Russell M. Church, and Warren H. Meck. 2013. "Bayesian Optimization of Time Perception" Trends in Cognitive Sciences 17: 556-64.

Smith, Jared G. et al. 2007. “The Effect of Parkinson's Disease on Time Estimation as a Function of Stimulus Duration Range and Modality" Brain and Cognition 64: 130-43. Sohn, Hansem, and Sang-Hun Lee. 2013. "Dichotomy in Perceptual Learning of Interval Timing: Calibration of Mean Accuracy and Precision Differ in Specificity and Time Course" Journal of Neurophysiology 109: 344-62.

Stelmach, George E., and Charles J. Worringham. 1988. "The Control of Bimanual Aiming Movements in Parkinson's Disease" Journal of Neurology, Neurosurgery, and Psychiatry 51: 223-31.

Tarantino, Vincenza et al. 2010. "The Time Course of Temporal Discrimination: An ERP Study" Clinical Neurophysiology 121: 43-52. 
Teki, Sundeep, Manon Grube, and Timothy D. Griffiths. 2012. "A Unified Model of Time Perception Accounts for Duration-Based and Beat-Based Timing Mechanisms" Frontiers in Integrative Neuroscience 5: 90.

Timmermann, Lars et al. 2003. "The Cerebral Oscillatory Network of Parkinsonian Resting Tremor" Brain 126: 199-212.

Timmermann, Lars et al. 2004. "Ten-Hertz Stimulation of Subthalamic Nucleus Deteriorates Motor Symptoms in Parkinson's Disease" Movement Disorders 19: 1328-33.

Treisman, Michel. 1963. "Temporal Discrimination and the Indifference Interval. Implications for a Model of the 'Internal Clock"' Psychological Monographs: General and Applied 77(13): 1-31.

Van Rijn, Hedderik et al. 2011. "Contingent Negative Variation and its Relation to Time Estimation: A Theoretical Evaluation" Frontiers in Integrative Neuroscience 5: 91.

Van Rijn, Hedderik, Bon-Mi Gu, and Warren H. Meck. 2014. "Dedicated Clock/TimingCircuit Theories of Time Perception and Timed Performance" Advances in Experimental Medicine and Biology 829: 75-99.

Van Wassenhove, Virginie et al. 2008. "Distortions of Subjective Time Perception Within and Across Senses" PLoS ONE 3(1): e1437.

Volkmann, J. et al. 1992. "Impairment of Temporal Organization of Speech in Basal Ganglia Diseases" Brain and Language 43: 386-99.

Volkow, Nora D. et al. 1998. "Association Between Decline in Brain Dopamine Activity With Age and Cognitive and Motor Impairment in Healthy Individuals" American Journal of Psychiatry 155: 344-9.

Wearden, J.H., and Helga Lejeune. 2008. "Scalar Properties in Human Timing: Conformity and Violations" The Quarterly Journal of Experimental Psychology 61: 569-587.

Wearden, J.H., and Rachel Grindrod. 2003. "Manipulating Decision Processes in the Human Scalar Timing System" Behavioural Processes 61: 47-56.

Williamson, Lauren L. et al. 2008. “'Speed' Warps Time: Methamphetamine's Interactive Roles in Drug Abuse, Habit Formation, and the Biological Clocks of Circadian and Interval Timing" Current Drug Abuse Reviews 1: 203-12.

Wing, Alan M., Steven Keele, and David I. Margolin. 1984. "Motor Disorder and the Timing of Repetitive Movements" Annals of The New York Academy of Sciences 423: 183-92.

Wise, Roy A. 2004. "Dopamine, Learning and Motivation" Nature Reviews Neuroscience $5: 483-94$

Wojtecki, Lars et al. 2006. "Frequency-Dependent Reciprocal Modulation of Verbal Fluency and Motor Functions in Subthalamic Deep Brain Stimulation" Archives of Neurology 63: 1273-6.

Wojtecki, Lars et al. 2011. "Modulation of Human Time Processing by Subthalamic Deep Brain Stimulation" PLoS ONE 6(9): e24589. 
Yin, Bin, and Andrew B. Troger. 2011. "Exploring the 4th Dimension: Hippocampus, Time, and Memory Revisited” Frontiers in Integrative Neuroscience 5: 36.

Yin, Bin, and Warren H. Meck. 2014. "Comparison of Interval Timing Behaviour in Mice Following Dorsal or Ventral Hippocampal Lesions with Mice Having $\delta$ Opioid Receptor Gene Deletion" Philosophical Transactions of the Royal Society - London B 369: 20120466.

Yin, Bin, Nicholas A. Lusk, and Warren H. Meck. 2015. "Interval-Timing Protocols and Their Relevancy to the Study of Temporal Cognition and Neurobehavioral Genetics" In Valter Tucci (ed.) Neuro-Phenome: Cutting-Edge Approaches and Technologies in Neurobehavioral Genetics. New York, NY: WIley-Blackwell, in press. 